\title{
The eigenvalues of the Laplacian for the homology of the Lie algebra corresponding to a poset
}

\author{
Iztok Hozo \\ Department of Mathematics \\ Indiana University Northwest \\ Gary, In 46408 \\ email: ihozo@iunhaw1.iun.indiana.edu \\ Submitted: April 6, 1995; Accepted: July 21, 1995.
}

\begin{abstract}
In this paper we study the spectral resolution of the Laplacian $\mathcal{L}$ of the Koszul complex of the Lie algebras corresponding to a certain class of posets.

Given a poset $P$ on the set $\{1,2, \ldots, n\}$, we define the nilpotent Lie algebra $L_{P}$ to be the span of all elementary matrices $z_{x, y}$, such that $x$ is less than $y$ in $P$. In this paper, we make a decisive step toward calculating the Lie algebra homology of $L_{P}$ in the case that the Hasse diagram of $P$ is a rooted tree.

We show that the Laplacian $\mathcal{L}$ simplifies significantly when the Lie algebra corresponds to a poset whose Hasse diagram is a tree. The main result of this paper determines the spectral resolutions of three commuting linear operators whose sum is the Laplacian $\mathcal{L}$ of the Koszul complex of $L_{P}$ in the case that the Hasse diagram is a rooted tree.

We show that these eigenvalues are integers, give a combinatorial indexing of these eigenvalues and describe the corresponding eigenspaces in representation-theoretic terms. The homology of $L_{P}$ is represented by the nullspace of $\mathcal{L}$, so in future work, these results should allow for the homology to be effectively computed.
\end{abstract}

AMS Classification Number: 17B56 (primary) 05E25 (secondary)

\section{Preliminaries}

\subsection{Definitions}

A partially ordered set $P$ (or poset, for short) is a set (which by abuse of notation we also call $P$ ), together with a binary relation denoted $\leq\left(\right.$ or $\leq_{P}$ when there is a possibility of confusion), satisfying the following three axioms:

1. For all $x \in P, x \leq x$. (reflexivity)

2. If $x \leq y$ and $y \leq x$, then $x=y$. (antisymmetry)

3. If $x \leq y$ and $y \leq z$, then $x \leq z$. (transitivity) 
A chain (or totally ordered set or linearly ordered set) is a poset in which any two elements are comparable. A subset $C$ of a poset $P$ is called a chain if $C$ is a chain when regarded as a subposet of $P$.

Definition 1.1 A poset $P$ is linear if for any two comparable elements $x, y \in P$, the interval $[x, y]$ is a chain, i.e., if every interval has the structure of a chain.

The length $l(C)$ of a finite chain $C$ is defined by $l(C)=|C|-1$.

\subsection{The homology of a poset}

The combinatorial approach to a homology theory for posets was developed by Rota [29], Farmer [8], Lakser [22], Mather [25], Crapo [5] and others (more references can be found in [33]). A systematic development of the relationship between the combinatorial and topological properties of posets was begun by K. Baclawski [1] and A. Björner [2] and continued by J. Walker [33].

Define the set $\mathcal{C}_{r}(P)$ to be the set of $0-1$ chains of length $r$ in the poset $P$. By abuse of notation we will use the same name for the complex vector space $C_{r}$ or $\mathcal{C}_{r}(P)$, with basis the set of $r$-chains. The $C_{r}$ 's are called chain spaces. The map $\partial_{r}: C_{r} \rightarrow C_{r-1}$, called the boundary map, is defined by:

$$
\partial_{r}\left(\hat{0}<x_{1}<\ldots<x_{r}<\hat{1}\right)=\sum_{i=1}^{r}(-1)^{i-1}\left(\hat{0}<x_{1}<\ldots<\widehat{x}_{i}<\ldots<x_{r}<\hat{1}\right)
$$

It is easy to check that:

\section{Lemma 1}

$$
\partial_{r-1} \circ \partial_{r}=0
$$

This allows us now to define the homology of a poset to be:

$$
H_{r}(P)=\operatorname{Ker}\left(\partial_{r}\right) / \operatorname{Im}\left(\partial_{r+1}\right)
$$

Later in this work we will talk about an operator, called the Laplacian of a complex, for which we need to identify the transpose of the boundary map. We are in fact transposing the matrix of the boundary map with respect to the basis of $r$-chains. In this case - the case of the poset homology, the transpose of the boundary map is not so difficult to evaluate.

Lemma 2 The transpose of the boundary operator (viewed as a linear map), is given by the following expression:

$$
\begin{aligned}
& \partial^{t}\left(\hat{0}<x_{1}<\ldots<x_{r}<\hat{1}\right) \\
& \quad=\sum_{i=0}^{r} \sum_{x_{i}<y<x_{i+1}}(-1)^{i}\left(\hat{0}<x_{1}<\ldots<x_{i}<y<x_{i+1}<\ldots<x_{r}<\hat{1}\right),
\end{aligned}
$$

where $x_{0}=\hat{0}$ and $x_{r+1}=\hat{1}$. 


\subsection{Lie Algebras}

In this section we will introduce some basic notions from the theory of Lie algebras, and the homology of Lie algebras.

We will always work over $\mathbb{C}$, the field of complex numbers.

Lie algebras arise "in nature" as vector spaces of linear transformations endowed with an operation which is in general neither commutative nor associative:

$$
[x, y]=x y-y x .
$$

It is possible to describe this kind of system abstractly in a few axioms.

Definition $1.2 A$ vector space $L$ over a field $\mathbb{C}$, with an operation $L \times L \rightarrow L$, denoted $(x, y) \rightarrow[x, y]$ and, called the bracket or commutator of $x$ and $y$, is a Lie algebra over $\mathbb{C}$ if the following axioms are satisfied:

The bracket operation is bilinear.

(L3) $[x,[y, z]]+[y,[z, x]]+[z,[x, y]]=0(x, y, z \in L)$.

Axiom (L3) is called Jacobi identity. The axioms (L1) and (L2) imply (L2'): $[x, y]=$ $-[y, x]$. In the field of complex numbers (L2') implies (L2).

\subsection{Homology of a Lie algebra}

Suppose $L$ is a Lie algebra and $A$ is a module over $L$. The space $\Gamma_{q}(L ; A)$ of $q$-dimensional chains of the Lie algebra $L$ with coefficients in $A$ is defined as $A \otimes \Lambda^{q} L$. The boundary operator $\partial=\partial_{q}: \Gamma_{q}(L ; A) \rightarrow \Gamma_{q-1}(L ; A)$ acts in accordance with the formula

$$
\begin{aligned}
\partial(a \otimes & \left.\left(x_{1} \wedge \ldots \wedge x_{q}\right)\right)= \\
= & \sum_{1 \leq s<t \leq q}(-1)^{s+t-1} a \otimes\left(\left[x_{s}, x_{t}\right] \wedge x_{1} \wedge \ldots \hat{x}_{s} \ldots \hat{x}_{t} \ldots \wedge x_{q}\right) \\
+ & \sum_{1 \leq s \leq q}(-1)^{s-1} x_{s} a \otimes\left(x_{1} \wedge \ldots \hat{x}_{s} \ldots \wedge x_{q}\right)
\end{aligned}
$$

\section{Lemma 3}

$$
\partial_{r-1} \partial_{r}=0
$$

The proof of this lemma is straightforward.

Let $\theta$ be the representation of $L$ on $A \otimes \Lambda^{q} L$. If $y \in L$, we have:

$$
\begin{aligned}
& \theta(y)\left(a \otimes x_{1} \wedge \ldots \wedge x_{q}\right) \\
& \quad=\left(y \cdot a \otimes x_{1} \wedge \ldots \wedge x_{q}\right)+\sum_{i}\left(a \otimes x_{1} \wedge \ldots \wedge\left[y, x_{i}\right] \wedge \ldots \wedge x_{q}\right)
\end{aligned}
$$

It is easy to check: 
Lemma 4 For $y \in L$ :

$$
\partial_{q} \circ \theta(y)=\theta(y) \circ \partial_{q}
$$

The homology of the complex $\left\{\Gamma_{q}(L ; A), \partial_{q}\right\}$ is referred to as the homology of the Lie algebra $L$ with coefficients in $A$ and denoted by $H_{q}(L ; A)$; if $A$ is the field of complex numbers viewed as a trivial $L$-module (as in our case), the second sum in the formula 1 vanishes. In this case the notations $\Gamma_{q}(L ; A)$ and $H_{q}(L ; A)$ are abbreviated to $\Gamma_{q}(L)$ and $H_{q}(L)$.

\subsection{The Laplacian operator}

Suppose that $\left\{\Gamma_{r}(L), \partial_{r}\right\}$ is a finite dimensional complex. We will first define an orthogonal inner product $\langle\cdot, \cdot\rangle$ on the product $\oplus \Gamma_{r}$, such that $\left\langle\Gamma_{r}, \Gamma_{s}\right\rangle=0$ whenever $r \neq s$. We will restrict our attention to the subspaces of the nilpotent Lie algebra $T_{n}(\mathbb{C})$ of all strictly upper triangular matrices over the complex numbers, with standard basis $\left\{z_{i, j}: 1 \leq i<j \leq n\right\}$, so we can define this product naturally:

Definition 1.3 Let $L$ be a Lie algebra, $L \subset T_{n}(\mathbb{C})$. Define an inner product for standard basis elements $v, w \in L$ by:

$$
\langle v, w\rangle=\left\{\begin{array}{rr}
1 & \text { if } v=w \\
0 & \text { otherwise } \\
0 & \text { if } v \text { and } w \text { have different exterior degrees }
\end{array}\right.
$$

Extend this to the exterior algebra, i.e., to the complexes mentioned above.

Definition 1.4 Suppose that $v=v_{1} \wedge \cdots \wedge v_{k}$ and $w=w_{1} \wedge \cdots \wedge w_{k}$. Then define the inner product:

$$
\langle v, w\rangle=\operatorname{det}\left(\left\langle v_{i}, w_{j}\right\rangle\right)_{1 \leq i, j \leq k}
$$

Note that this can be written also as

$$
\langle v, w\rangle=\sum_{\sigma \in S_{n}} \operatorname{sgn}(\sigma) \prod_{i}\left\langle v_{i}, w_{\sigma(i)}\right\rangle=\left\{\begin{array}{lr}
\operatorname{sgn}(\sigma) & \text { iff } v_{i}=w_{\sigma(i)} \text { for all } i \\
0 & \text { otherwise }
\end{array}\right.
$$

In other words, the product of two pure wedges of basis elements is nonzero if and only if two pure wedges differ only in the order of the elements, and in that case, the product is just the sign of the permutation that changes one into another.

Define $\delta_{r}$ mapping $\Gamma_{r}$ into $\Gamma_{r+1}$ by

$$
\left\langle\delta_{r} v, w\right\rangle=\left\langle v, \partial_{r+1} w\right\rangle
$$

over all $v \in \Gamma_{r}$, and all $w \in \Gamma_{r+1}$. It is enough to calculate $\delta$ on pure wedges (as in our definitions), since the inner product and $\delta$ are both linear functions. 
Lemma 5 The map $\delta$ is given by

$$
\begin{aligned}
& \delta_{r}\left(z_{x_{1}, y_{1}} \wedge z_{x_{2}, y_{2}} \wedge \ldots \wedge z_{x_{r}, y_{r}}\right) \\
& \quad=\sum_{s=1}^{r}(-1)^{s-1} \sum_{x_{s}<l<y_{s}} z_{x_{1}, y_{1}} \wedge \ldots \wedge z_{x_{s}, l} \wedge z_{l, y_{s}} \wedge \ldots \wedge z_{x_{r}, y_{r}}
\end{aligned}
$$

Note: It is easy to check that $\delta_{r+1} \delta_{r}=0$, thus $\delta_{*}$ defines a coboundary operator, and so we can define the cohomology to be

$$
H^{r}(L)=\operatorname{Ker}\left(\delta_{r}\right) / \operatorname{Im}\left(\delta_{r-1}\right)
$$

Proof: But to prove that, it is enough to show that the coefficient of the pure wedge $z_{x_{1}, y_{1}} \wedge z_{x_{2}, y_{2}} \wedge \ldots \wedge z_{x_{r}, y_{r}}$ in $\partial\left(z_{x_{1}, y_{1}} \wedge \cdots \wedge z_{x_{s}, l} \wedge z_{l, y_{s}} \wedge \cdots \wedge z_{x_{r}, y_{r}}\right)$ is $(-1)^{s-1}$ for any $l \in\left(x_{s}, y_{s}\right)$, i.e.,

$$
\begin{aligned}
& \partial\left(z_{x_{1}, y_{1}} \wedge \ldots \wedge z_{x_{s}, l} \wedge z_{l, y_{s}} \wedge \ldots \wedge z_{x_{r}, y_{r}}\right) \\
& \quad=\ldots+(-1)^{s-1}\left(z_{x_{1}, y_{1}} \wedge z_{x_{2}, y_{2}} \wedge \ldots \wedge z_{x_{r}, y_{r}}\right)+\ldots
\end{aligned}
$$

and this is not difficult by the definition of $\partial$.

Note that we can change the order of the elements in the pure wedges, and obtain a slightly different form for $\delta$ :

$$
\begin{aligned}
& \delta_{r}\left(z_{x_{1}, y_{1}} \wedge z_{x_{2}, y_{2}} \wedge \ldots \wedge z_{x_{r}, y_{r}}\right) \\
& \quad=\sum_{s=1}^{r}(-1)^{s-1} \sum_{x_{s}<l<y_{s}} z_{x_{1}, y_{1}} \wedge \ldots \wedge z_{x_{s}, l} \wedge z_{l, y_{s}} \wedge \ldots \wedge z_{x_{r}, y_{r}} \\
& \quad=\sum_{m} \sum_{x_{m}<l<y_{m}}\left(z_{x_{m}, l} \wedge z_{x_{1}, y_{1}} \wedge \cdots \wedge z_{l, y_{m}} \wedge \cdots \wedge z_{x_{k}, y_{k}}\right)
\end{aligned}
$$

This is the form for the $\delta=\partial^{t}$ we will use.

Definition 1.5 Define the Laplacian operator $L_{r}: \Gamma_{r} \rightarrow \Gamma_{r}$ by

$$
L_{r}=\delta_{r-1} \partial_{r}+\partial_{r+1} \delta_{r}
$$

Theorem 6 (Kostant, [19] ) Let $B=\left\{\beta_{1}, \ldots, \beta_{d}\right\}$ be a basis for $\operatorname{Ker}\left(L_{r}\right)$. Then $B$ is simultaneously a complete set of representatives of $H^{r}(L)$ and $H_{r}(L)$. In particular $\operatorname{dim}\left(H^{r}(L)\right)=\operatorname{dim}\left(H_{r}(L)\right)=\operatorname{dim}\left(\operatorname{Ker}\left(L_{r}\right)\right)$.

Sometimes, the Laplacian $L_{r}$ will turn out to be very simple. In these cases, Theorem 6 is a very efficient method for evaluating the homology and cohomology of a Lie algebra. One famous result obtained in this way is given by Kostant [19]. 


\subsection{Kostant's Theorem}

We need some preliminary definitions. Suppose $\mathcal{G}$ is a semisimple Lie algebra, with the root system $R$, whose basis is $\Delta$. Thus $\mathcal{G}=H \oplus\left(\oplus_{\alpha \in R}\left\langle z_{\alpha}\right\rangle\right)$, where $H$ is the torus. Suppose that $S \subset \Delta$, and let $R_{S}$ be the set of roots in the $\mathbb{Z}$ (integer) module spanned by elements of $S$. Define $\mathcal{G}_{\mathcal{S}}$ to be $\mathcal{G}_{\mathcal{S}}=H \oplus\left\langle z_{\alpha}: \alpha \in R_{S}\right\rangle$. Define a $\mathcal{G}_{S}$ module $N_{S}$ to be $N_{S}=\left\langle z_{\alpha}: \alpha \in R^{+} \backslash R_{S}^{+}\right\rangle$.

We will state a couple of facts without proof:

- $N_{S}$ is a nilpotent subalgebra of $\mathcal{G}$.

- Let $W$ be a $\mathcal{G}$-module. Then $W$ is also a $N_{S}$-module and a $\mathcal{G}_{\mathcal{S}}$-module.

- Thus we can compute $H\left(N_{S} ; W^{\mu}\right)$ as $\mathcal{G}_{\mathcal{S}}$-module, where $W^{\mu}$ is an irreducible $\mathcal{G}$ module. Kostant used the Laplacian operator to prove the following theorem:

Theorem 7 (Kostant, Theorem 5.7,[19]) Let $\lambda$ be a dominant weight for $\mathcal{G}$, and let $\mu$ be a minimal weight for $\mathcal{G}_{\mathcal{S}}$. Let $V$ be a $\mathcal{G}_{\mathcal{S}}$-invariant subspace of $W^{\lambda} \otimes \bigwedge^{r} N_{S}$ isomorphic to the $\mathcal{G}_{\mathcal{S}}$-irreducible (indexed by $\mu$ ) with minimal weight $\mu$.

- The Laplacian $L=\delta \partial+\partial \delta$ preserves $V$.

- Then, $\left.L\right|_{V}$ is a scalar, and the scalar is given by

$$
\frac{1}{2}\left(|\rho+\lambda|^{2}-|\rho-\mu|^{2}\right)
$$

where $\rho$ is half of the sum of the positive roots of $\mathcal{G}$.

\subsection{The Lie Algebra corresponding to a Poset}

Definition 1.6 A standard labeling of the poset $P$ is a total ordering of the elements of $P$ such that whenever $x<_{P} y, x$ precedes $y$ in that total ordering.

Since $P$ is a partial order, i.e. transitive, there always is such labeling. Fix a standard labeling of the poset $P$.

We can define a Lie algebra $L_{P}$ corresponding to the poset $P$ in the following way. First, for every relation $x<_{P} y$ in the poset $P$, i.e., for every two elements $x, y \in P$ such that $x<_{P} y$ we can define the matrix $z_{x, y}$ having all entries equal to zero, except for exactly one entry equal to 1 , namely the entry at the position $x, y$ in the standard labeling of the poset $P$.

All matrices $z_{x, y}$ are strictly upper triangular because of our labeling. So $L_{P}$ is a subalgebra of $T_{n}$. The Lie algebras $L_{P}$ obtained from distinct labellings are isomorphic the labeling only specifies embedding of $L_{P}$ in the $n \times n$ matrices. 


\section{The Formula for Laplacian of a Linear Poset}

In this section we will present a significant simplification of the Lie algebra Laplacian in the case of linear posets. That will allow us to prove our main result on the eigenvalues of those Laplacians.

\subsection{Simplification}

Recall the Lie algebra boundary map:

$$
\begin{aligned}
& \partial\left(z_{x_{1}, y_{1}} \wedge \ldots \wedge z_{x_{k}, y_{k}}\right) \\
& \quad=\sum_{i<j}(-1)^{i+j-1}\left[z_{x_{i}, y_{i}}, z_{x_{j}, y_{j}}\right] \wedge z_{x_{1}, y_{1}} \wedge \ldots \wedge \widehat{z_{x_{i}, y_{i}}} \wedge \ldots \wedge \widehat{z_{x_{j}, y_{j}}} \wedge \ldots \wedge z_{x_{k}, y_{k}}
\end{aligned}
$$

The transpose, $\partial^{t}$, is given by the following formula:

$$
\begin{aligned}
& \partial_{r}^{t}\left(z_{x_{1}, y_{1}} \wedge z_{x_{2}, y_{2}} \wedge \ldots \wedge z_{x_{r}, y_{r}}\right) \\
& \quad=\sum_{s=1}^{r}(-1)^{s-1} \sum_{x_{s}<l<y_{s}} z_{x_{1}, y_{1}} \wedge \ldots \wedge z_{x_{s}, l} \wedge z_{l, y_{s}} \wedge \ldots \wedge z_{x_{r}, y_{r}} \\
& \quad=\sum_{m} \sum_{x_{m}<l<y_{m}}\left(z_{x_{m}, l} \wedge z_{x_{1}, y_{1}} \wedge \cdots \wedge z_{l, y_{m}} \wedge \cdots \wedge z_{x_{k}, y_{k}}\right)
\end{aligned}
$$

To compute the action of $L$ on a basis vector $z_{x_{1}, y_{1}} \wedge \cdots \wedge z_{x_{k}, y_{k}}$ of $\Gamma_{k}\left(L_{P}\right)$ we begin with the action of $\partial \partial^{t}$. We have,

$$
\begin{aligned}
\partial \partial^{t}\left(z_{x_{1}, y_{1}} \wedge \cdots \wedge z_{x_{k}, y_{k}}\right) & \\
= & \sum_{m} \sum_{x_{m}<l<y_{m}} \partial\left(z_{x_{m}, l} \wedge z_{x_{1}, y_{1}} \wedge \cdots \wedge z_{l, y_{m}} \wedge \cdots \wedge z_{x_{k}, y_{k}}\right) \\
= & \sum_{i<j} \sum_{m \neq i, j} \sum_{x_{m}<l<y_{m}}(-1)^{i+1+j}\left(\left[z_{x_{i}, y_{i}}, z_{x_{j}, y_{j}}\right] \wedge z_{x_{m}, l} \wedge z_{x_{1}, y_{1}} \wedge \ldots\right. \\
& \left.\ldots \wedge \widehat{z_{x_{i}, y_{i}}} \wedge \cdots \wedge z_{l, y_{m}} \wedge \cdots \wedge \widehat{z_{x_{j}, y_{j}}} \wedge \cdots \wedge z_{x_{k}, y_{k}}\right) \\
+ & \sum_{m} \sum_{j \neq m} \sum_{x_{m}<l<y_{m}}(-1)^{1+j+1-1}\left(\left[z_{x_{m}, l}, z_{x_{j}, y_{j}}\right] \wedge z_{x_{1}, y_{1}} \wedge \ldots\right. \\
+ & \sum_{i<m} \widehat{\sum_{x_{m}<l<y_{m}}}(-1)^{i+1+m+1-1}\left(\left[z_{x_{i}, y_{i}}, z_{l, y_{m}}\right] \wedge z_{x_{m}, l} \wedge z_{x_{1}, y_{1}} \wedge \ldots\right. \\
& \left.\ldots \wedge \widehat{z_{x_{i}, y_{i}}} \wedge \cdots \wedge \widehat{z_{x_{m}, y_{m}}} \wedge \cdots \wedge z_{x_{k}, y_{k}}\right) \\
+ & \sum_{m<j} \widehat{\sum_{x_{m}<l<y_{m}}}(-1)^{m+1+j+1-1}\left(\left[z_{l, y_{m}}, z_{x_{j}, y_{j}}\right] \wedge z_{x_{m}, l} \wedge z_{x_{1}, y_{1}} \wedge \ldots\right. \\
& \left.\ldots \wedge \widehat{z_{x_{m}, y_{m}}} \wedge \cdots \wedge \widehat{z_{x_{j}, y_{j}}} \wedge \cdots \wedge z_{x_{k}, y_{k}}\right) \\
+ & \sum_{m=1}^{k}\left|\left(\widehat{x_{m}, y_{m}}\right)\right|\left(z_{x_{1}, y_{1}} \wedge \cdots \wedge z_{x_{k}, y_{k}}\right)
\end{aligned}
$$


which is equal to:

$$
\begin{aligned}
= & \sum_{i<j} \sum_{m \neq i, j} \sum_{x_{m}<l<y_{m}}(-1)^{i+j-1}\left(\left[z_{x_{i}, y_{i}}, z_{x_{j}, y_{j}}\right] \wedge z_{x_{m}, l} \wedge z_{x_{1}, y_{1}} \wedge \ldots\right. \\
& \left.\ldots \wedge \widehat{z_{x_{i}, y_{i}}} \wedge \cdots \wedge z_{l, y_{m}} \wedge \cdots \wedge \widehat{z_{x_{j}, y_{j}}} \wedge \cdots \wedge z_{x_{k}, y_{k}}\right) \\
+ & \sum_{i<m} \sum_{x_{m}<l<y_{m}}(-1)^{i+1}\left(\left[z_{x_{m}, l}, z_{x_{i}, y_{i}}\right] \wedge z_{x_{1}, y_{1}} \wedge \ldots\right. \\
& \left.\ldots \wedge \widehat{z_{x_{i}, y_{i}}} \wedge \cdots \wedge z_{l, y_{m}} \wedge \cdots \wedge z_{x_{k}, y_{k}}\right) \\
& \sum_{m<j} \sum_{x_{m}<l<y_{m}}(-1)^{j+1}\left(\left[z_{x_{m}, l}, z_{x_{j}, y_{j}}\right] \wedge z_{x_{1}, y_{1}} \wedge \ldots\right. \\
& \left.\ldots \wedge \widehat{z_{l, y_{m}}} \wedge \cdots \wedge \widehat{z_{x_{j}, y_{j}}} \wedge \cdots \wedge z_{x_{k}, y_{k}}\right) \\
& \sum_{i<m} \widehat{\sum_{x_{m}<l<y_{m}}}(-1)^{i+m+1}\left(\left[z_{x_{i}, y_{i}}, z_{l, y_{m}}\right] \wedge z_{x_{m}, l} \wedge z_{x_{1}, y_{1}} \wedge \ldots\right. \\
& \left.\ldots \wedge \widehat{z_{x_{i}, y_{i}}} \wedge \cdots \wedge \widehat{z_{x_{m}, y_{m}}} \wedge \cdots \wedge z_{x_{k}, y_{k}}\right) \\
+ & \sum_{m<j} \widehat{\sum_{x_{m}<l<y_{m}}}(-1)^{m+j+1}\left(\left[z_{l, y_{m}}, z_{x_{j}, y_{j}}\right] \wedge z_{x_{m}, l} \wedge z_{x_{1}, y_{1}} \wedge \ldots\right. \\
& \left.\ldots \wedge \widehat{z_{x_{m}, y_{m}}} \wedge \cdots \wedge \widehat{z_{x_{j}, y_{j}}} \wedge \cdots \wedge z_{x_{k}, y_{k}}\right) \\
+ & \left.\sum_{m=1}^{k} \mid \widehat{\left(x_{m}, y_{m}\right.}\right) \mid\left(z_{x_{1}, y_{1}} \wedge \cdots \wedge z_{x_{k}, y_{k}}\right)
\end{aligned}
$$

Now use the definition of bracket in this Lie algebra:

$$
\left[z_{x_{i}, y_{i}}, z_{x_{j}, y_{j}}\right]=\delta_{y_{i}, x_{j}} z_{x_{i}, y_{j}}-\delta_{x_{i}, y_{j}} z_{x_{j}, y_{i}}
$$

and we have the following:

$$
\begin{aligned}
\partial \partial^{t}\left(z_{x_{1}, y_{1}} \wedge \cdots \wedge z_{x_{k}, y_{k}}\right) & \\
= & \sum_{i<j} \sum_{m \neq i, j} \sum_{x_{m}<l<y_{m}}(-1)^{i+j-1}\left(\left[z_{x_{i}, y_{i}}, z_{x_{j}, y_{j}}\right] \wedge z_{x_{m}, l} \wedge z_{x_{1}, y_{1}} \wedge \cdots \wedge \widehat{z_{x_{i}, y_{i}}} \wedge \ldots\right. \\
& \left.\quad \cdots \wedge z_{l, y_{m}} \wedge \cdots \wedge \widehat{z_{x_{j}, y_{j}}} \wedge \cdots \wedge z_{x_{k}, y_{k}}\right) \\
& +\sum_{i<m} \sum_{x_{m}<l<y_{m}} \delta_{l, x_{i}}\left(z_{x_{1}, y_{1}} \wedge \cdots \wedge z_{x_{m}, y_{i}} \wedge \cdots \wedge z_{l, y_{m}} \wedge \cdots \wedge z_{x_{k}, y_{k}}\right) \\
& -\delta_{x_{m}, y_{i}}\left(z_{x_{1}, y_{1}} \wedge \cdots \wedge z_{x_{i}, l} \wedge \cdots \wedge z_{l, y_{m}} \wedge \cdots \wedge z_{x_{k}, y_{k}}\right) \\
& +\sum_{m<j} \sum_{x_{m}<l<y_{m}} \delta_{l, x_{j}}\left(z_{x_{1}, y_{1}} \wedge \cdots \wedge z_{l, y_{m}} \wedge \cdots \wedge z_{x_{m}, y_{j}} \wedge \cdots \wedge z_{x_{k}, y_{k}}\right) \\
& -\delta_{x_{m}, y_{j}}\left(z_{x_{1}, y_{1}} \wedge \cdots \wedge z_{l, y_{m}} \wedge \cdots \wedge z_{x_{j}, l} \wedge \cdots \wedge z_{x_{k}, y_{k}}\right) \\
+ & \sum_{i<m} \sum_{x_{m}<l<y_{m}} \delta_{l, y_{i}}\left(z_{x_{1}, y_{1}} \wedge \cdots \wedge z_{x_{i}, y_{j}} \wedge \cdots \wedge z_{x_{m}, l} \wedge \cdots \wedge z_{x_{k}, y_{k}}\right) \\
- & \delta_{x_{i}, y_{m}}\left(z_{x_{1}, y_{1}} \wedge \cdots \wedge z_{l, y_{i}} \wedge \cdots \wedge z_{x_{m}, l} \wedge \cdots \wedge z_{x_{k}, y_{k}}\right)
\end{aligned}
$$




$$
\begin{aligned}
& +\sum_{m<j} \sum_{x_{m}<l<y_{m}} \delta_{l, y_{j}}\left(z_{x_{1}, y_{1}} \wedge \cdots \wedge z_{x_{m}, l} \wedge \cdots \wedge z_{x_{j}, y_{m}} \wedge \cdots \wedge z_{x_{k}, y_{k}}\right) \\
& -\delta_{x_{j}, y_{m}}\left(z_{x_{1}, y_{1}} \wedge \cdots \wedge z_{x_{m}, l} \wedge \cdots \wedge z_{l, y_{j}} \wedge \cdots \wedge z_{x_{k}, y_{k}}\right) \\
& +\sum_{m=1}^{k}\left|\left(x_{m}, y_{m}\right)\right|\left(z_{x_{1}, y_{1}} \wedge \cdots \wedge z_{x_{k}, y_{k}}\right)
\end{aligned}
$$

Note that every sum over $x_{m}<l<y_{m}$ which has an occurrence of $\delta_{l, *}$ has only one summand if $*$ really is between $x_{m}$ and $y_{m}$, and is zero otherwise. We will use the symbol $\chi$ for denoting the truth of some statement, i.e.,

$$
\chi(*)= \begin{cases}1, & \text { if } * \text { is true } \\ 0, & \text { if } * \text { is false }\end{cases}
$$

We label some of the resulting sums:

$$
\begin{aligned}
\partial \partial^{t}\left(z_{x_{1}, y_{1}} \wedge \cdots \wedge z_{x_{k}, y_{k}}\right) & =\sum_{i<j} \sum_{m \neq i, j}(-1)^{i+j-1}\left(\left[z_{x_{i}, y_{i}}, z_{x_{j}, y_{j}}\right] \wedge z_{x_{m}, l} \wedge z_{x_{1}, y_{1}} \wedge \ldots\right. \\
& \left.=\ldots \wedge \widehat{z_{x_{i}, y_{i}}} \wedge \cdots \wedge z_{l, y_{m}} \wedge \cdots \wedge \widehat{z_{x_{j}, y_{j}}} \wedge \cdots \wedge z_{x_{k}, y_{k}}\right) \\
& -\sum_{i<j} \chi\left(x_{j}<x_{i}<y_{j}\right)\left(z_{x_{1}, y_{1}} \wedge \cdots \wedge z_{x_{i}, y_{j}} \wedge \cdots \wedge z_{x_{j}, y_{i}} \wedge \cdots \wedge z_{x_{k}, y_{k}}\right) \\
& -\sum_{i<j} \sum_{x_{j}<l<y_{j}} \delta_{x_{j}, y_{i}}\left(z_{x_{1}, y_{1}} \wedge \cdots \wedge z_{x_{i}, l} \wedge \cdots \wedge z_{l, y_{j}} \wedge \cdots \wedge z_{x_{k}, y_{k}}\right) \\
& -\sum_{i<j} \chi\left(x_{i}<x_{j}<y_{i}\right)\left(z_{x_{1}, y_{1}} \wedge \cdots \wedge z_{x_{i}, y_{j}} \wedge \cdots \wedge z_{x_{j}, y_{i}} \wedge \cdots \wedge z_{x_{k}, y_{k}}\right) \\
& -\sum_{i<j} \sum_{x_{i}<l<y_{i}} \delta_{x_{i}, y_{j}}\left(z_{x_{1}, y_{1}} \wedge \cdots \wedge z_{l, y_{i}} \wedge \cdots \wedge z_{x_{j}, l} \wedge \cdots \wedge z_{x_{k}, y_{k}}\right) \\
& +\sum_{i<j} \chi\left(x_{j}<y_{i}<y_{j}\right)\left(z_{x_{1}, y_{1}} \wedge \cdots \wedge z_{x_{i}, y_{j}} \wedge \cdots \wedge z_{x_{j}, y_{i}} \wedge \cdots \wedge z_{x_{k}, y_{k}}\right) \\
& -\sum_{i<j} \sum_{x_{j}<l<y_{j}} \delta_{x_{i}, y_{j}}\left(z_{x_{1}, y_{1}} \wedge \cdots \wedge z_{l, y_{i}} \wedge \cdots \wedge z_{x_{j}, l} \wedge \cdots \wedge z_{x_{k}, y_{k}}\right) \\
& -\sum_{i<j} \sum_{x_{i}<l<y_{i}} \delta_{x_{j}, y_{i}}\left(z_{x_{1}, y_{1}} \wedge \cdots \wedge z_{x_{i}, l} \wedge \cdots \wedge z_{l, y_{j}} \wedge \cdots \wedge z_{x_{k}, y_{k}}\right) \\
& +\sum_{i<j} \chi\left(x_{i}<y_{j}<y_{i}\right)\left(z_{x_{1}, y_{1}} \wedge \cdots \wedge z_{x_{i}, y_{j}} \wedge \cdots \wedge z_{x_{j}, y_{i}} \wedge \cdots \wedge z_{x_{k}, y_{k}}\right) \\
& \sum_{m=1}^{k}\left|\left(x_{m}, y_{m}\right)\right|\left(z_{x_{1}, y_{1}} \wedge \cdots \wedge z_{x_{k}, y_{k}}\right)
\end{aligned}
$$

On the other hand: 


$$
\begin{aligned}
& \partial^{t} \partial\left(z_{x_{1}, y_{1}} \wedge \cdots \wedge z_{x_{k}, y_{k}}\right) \\
& =\sum_{i<j}(-1)^{i+j-1} \partial^{t}\left(\left[z_{x_{i}, y_{i}}, z_{x_{j}, y_{j}}\right] \wedge z_{x_{1}, y_{1}} \cdots \wedge \widehat{z_{x_{i}, y_{i}}} \wedge \cdots \wedge \widehat{z_{x_{j}, y_{j}}} \wedge \cdots \wedge z_{x_{k}, y_{k}}\right) \\
& =\sum_{i<j} \sum_{m \neq i, j} \sum_{x_{m}<l<y_{m}}(-1)^{i+j-1}\left(z_{x_{m}, l} \wedge\left[z_{x_{i}, y_{i}}, z_{x_{j}, y_{j}}\right] \wedge z_{x_{1}, y_{1}} \wedge \ldots\right. \\
& \left.\cdots \wedge \widehat{z_{x_{i}, y_{i}}} \wedge \cdots \wedge z_{l, y_{m}} \wedge \cdots \wedge \widehat{z_{x_{j}, y_{j}}} \wedge \cdots \wedge z_{x_{k}, y_{k}}\right) \\
& +\sum_{i<j} \sum_{x_{m}<l<y_{m}}(-1)^{i+j-1} \delta_{x_{j}, y_{i}}\left(z_{x_{i}, l} \wedge z_{l, y_{j}} \wedge z_{x_{1}, y_{1}} \wedge \ldots\right. \\
& \left.\cdots \wedge \widehat{z_{x_{i}, y_{i}}} \wedge \cdots \wedge \widehat{z_{x_{j}, y_{j}}} \wedge \cdots \wedge z_{x_{k}, y_{k}}\right) \\
& -\sum_{i<j} \sum_{x_{m}<l<y_{m}}(-1)^{i+j-1} \delta_{x_{i}, y_{j}}\left(z_{x_{j}, l} \wedge z_{l, y_{i}} \wedge z_{x_{1}, y_{1}} \wedge \ldots\right. \\
& \left.\cdots \wedge \widehat{z_{x_{i}, y_{i}}} \wedge \cdots \wedge \widehat{z_{x_{j}, y_{j}}} \wedge \cdots \wedge z_{x_{k}, y_{k}}\right)
\end{aligned}
$$

Now use the fact that we are dealing with a linear poset. This implies that for every interval $\left(x_{m}, y_{m}\right)$ and every $l, x_{m}<l<y_{m}$ we have

$$
\left(x_{m}, y_{m}\right)=\left(x_{m}, l\right) \cup\{l\} \cup\left(l, y_{m}\right)
$$

Hence

$$
\begin{aligned}
\partial^{t} \partial & \left(z_{x_{1}, y_{1}} \wedge \cdots \wedge z_{x_{k}, y_{k}}\right) \\
& =\sum_{i<j} \sum_{m \neq i, j} \sum_{x_{m}<l<y_{m}}(-1)^{i+j-1}\left(z_{x_{m}, l} \wedge\left[z_{x_{i}, y_{i}}, z_{x_{j}, y_{j}}\right] \wedge z_{x_{1}, y_{1}} \wedge \ldots\right. \\
& +\sum_{i<j} \sum_{x_{i}<l<y_{i}} \delta_{x_{j}, y_{i}}\left(z_{x_{1}, y_{1}} \wedge \cdots \wedge z_{x_{i}, l} \wedge \cdots \wedge z_{l, y_{j}} \wedge \cdots \wedge z_{x_{k}, y_{k}}\right) \\
& +\sum_{i<j} \sum_{l=x_{j}=y_{i}} \delta_{x_{j}, y_{i}}\left(z_{x_{1}, y_{1}} \wedge \cdots \wedge z_{x_{i}, l} \wedge \cdots \wedge z_{l, y_{j}} \wedge \cdots \wedge z_{x_{k}, y_{k}}\right) \\
& +\sum_{i<j} \sum_{x_{j}<l<y_{j}} \delta_{x_{j}, y_{i}}\left(z_{x_{1}, y_{1}} \wedge \cdots \wedge z_{x_{i}, l} \wedge \cdots \wedge z_{l, y_{j}} \wedge \cdots \wedge z_{x_{k}, y_{k}}\right) \\
& +\sum_{i<j} \sum_{x_{j}<l<y_{j}} \delta_{x_{i}, y_{j}}\left(z_{x_{1}, y_{1}} \wedge \cdots \wedge z_{l, y_{i}} \wedge \cdots \wedge z_{x_{j}, l} \wedge \cdots \wedge z_{x_{k}, y_{k}}\right) \\
& +\sum_{i<j} \sum_{l=x_{i}=y_{j}} \delta_{x_{i}, y_{j}}\left(z_{x_{1}, y_{1}} \wedge \cdots \wedge z_{l, y_{i}} \wedge \cdots \wedge z_{x_{j}, l} \wedge \cdots \wedge z_{x_{k}, y_{k}}\right) \\
& +\sum_{i<j} \sum_{x_{i}<l<y_{i}} \delta_{x_{i}, y_{j}}\left(z_{x_{1}, y_{1}} \wedge \cdots \wedge z_{l, y_{i}} \wedge \cdots \wedge z_{x_{j}, l} \wedge \cdots \wedge z_{x_{k}, y_{k}}\right)
\end{aligned}
$$

Then we have : 


$$
\begin{aligned}
(9)+(3) & =0 \\
(10)+(7) & =0 \\
(11)+(4) & =0 \\
(12)+(6) & =0 \\
(13)+(5) & =0
\end{aligned}
$$

After these cancellations we obtain the following expression for the action of the Laplacian $L$ :

$$
\begin{aligned}
& L\left(z_{x_{1}, y_{1}} \wedge \cdots \wedge z_{x_{k}, y_{k}}\right)=\left(\partial \partial^{t}+\partial^{t} \partial\right)\left(z_{x_{1}, y_{1}} \wedge \cdots \wedge z_{x_{k}, y_{k}}\right) \\
& \quad=\sum_{m=1}^{k}\left|\left(x_{m}, y_{m}\right)\right|\left(z_{x_{1}, y_{1}} \wedge \cdots \wedge z_{x_{k}, y_{k}}\right) \\
& \quad+\sum_{i<j}\left(\delta_{x_{i}, y_{j}}+\delta_{x_{j}, y_{i}}\right)\left(z_{x_{1}, y_{1}} \wedge \cdots \wedge z_{x_{k}, y_{k}}\right) \\
& \quad+\sum_{i<j} \chi\left(x_{i}<y_{j}<y_{i}\right)\left(z_{x_{1}, y_{1}} \wedge \cdots \wedge z_{x_{i}, y_{j}} \wedge \cdots \wedge z_{x_{j}, y_{i}} \wedge \cdots \wedge z_{x_{k}, y_{k}}\right) \\
& \quad+\sum_{i<j} \chi\left(x_{j}<y_{i}<y_{j}\right)\left(z_{x_{1}, y_{1}} \wedge \cdots \wedge z_{x_{i}, y_{j}} \wedge \cdots \wedge z_{x_{j}, y_{i}} \wedge \cdots \wedge z_{x_{k}, y_{k}}\right) \\
& \quad-\sum_{i<j} \chi\left(x_{j}<x_{i}<y_{j}\right)\left(z_{x_{1}, y_{1}} \wedge \cdots \wedge z_{x_{i}, y_{j}} \wedge \cdots \wedge z_{x_{j}, y_{i}} \wedge \cdots \wedge z_{x_{k}, y_{k}}\right) \\
& \quad-\sum_{i<j} \chi\left(x_{i}<x_{j}<y_{i}\right)\left(z_{x_{1}, y_{1}} \wedge \cdots \wedge z_{x_{i}, y_{j}} \wedge \cdots \wedge z_{x_{j}, y_{i}} \wedge \cdots \wedge z_{x_{k}, y_{k}}\right)
\end{aligned}
$$

\subsection{The Formula}

To further simplify our expressions we will introduce some notation. Define

$$
\begin{aligned}
\zeta & =z_{x_{1}, y_{1}} \wedge \cdots \wedge z_{x_{k}, y_{k}} \\
\zeta_{i, j} & =z_{x_{1}, y_{1}} \wedge \cdots \wedge z_{x_{i}, y_{j}} \wedge \cdots \wedge z_{x_{j}, y_{i}} \wedge \cdots \wedge z_{x_{k}, y_{k}} \\
w(\zeta) & =\sum_{m=1}^{k}\left|\left(x_{m}, y_{m}\right)\right| \\
\Delta(\zeta) & =\sum_{i<j}\left(\delta_{x_{i}, y_{j}}+\delta_{x_{j}, y_{i}}\right)=\sum_{i, j} \delta_{x_{i}, y_{j}}
\end{aligned}
$$

Thus, we can reformulate the calculations from the previous section into:

Theorem 8 (The Formula) Let $P$ be a linear poset and let $L_{P}$ be the corresponding Lie algebra. The action of the Laplacian $L$ on an element

$$
\zeta=z_{x_{1}, y_{1}} \wedge z_{x_{2}, y_{2}} \wedge \cdots \wedge z_{x_{k}, y_{k}}
$$


is given by the following formula:

$$
\begin{aligned}
& L(\zeta)=(w(\zeta)+\Delta(\zeta)) \zeta \\
& \quad+\sum_{i<j}\left(\chi\left(x_{i}<y_{j}<y_{i}\right)+\chi\left(x_{j}<y_{i}<y_{j}\right)-\chi\left(x_{j}<x_{i}<y_{j}\right)-\chi\left(x_{i}<x_{j}<y_{i}\right)\right) \zeta_{i, j}
\end{aligned}
$$

Note that $\zeta_{i, j}$ is obtained from $\zeta$ by transposing a comparable pair of $y$ 's or a comparable pair of $x$ 's.

\section{Linear poset with a $\hat{0}$}

Suppose now that the poset $P$ has a $\hat{0}$, the minimum element. That is the assumption under which we will work in the future. In that case, we can further simplify our notation:

\section{Lemma 9}

$$
\begin{aligned}
& L(\zeta)=(w(\zeta)+\Delta(\zeta)) \zeta \\
& \quad+\sum_{i<j}\left(\chi\left(x_{i}<y_{j}<y_{i}\right)+\chi\left(x_{j}<y_{i}<y_{j}\right)-\chi\left(x_{j}<x_{i}<y_{j}\right)-\chi\left(x_{i}<x_{j}<y_{i}\right)\right) \zeta_{i, j} \\
& =(w(\zeta)+\Delta(\zeta)) \zeta+\sum_{y_{i}<P y_{j}} \zeta_{i, j}-\sum_{x_{i}<P x_{j}} \zeta_{i, j}
\end{aligned}
$$

Proof: We need to prove that we can write

$$
\chi\left(y_{i}<y_{j}\right)+\chi\left(y_{j}<y_{i}\right)-\chi\left(x_{i}<x_{j}\right)-\chi\left(x_{j}<x_{i}\right)
$$

instead of

$$
\chi\left(x_{i}<y_{j}<y_{i}\right)+\chi\left(x_{j}<y_{i}<y_{j}\right)-\chi\left(x_{j}<x_{i}<y_{j}\right)-\chi\left(x_{i}<x_{j}<y_{i}\right)
$$

in the expression for the Laplacian above.

Let $y_{i}$ and $y_{j}$ be two comparable distinct $y$ 's. Without loss of generality, assume that $y_{i}<y_{j}$. Thus $x_{i}<y_{i}<y_{j}$. The existence of $\hat{0}$ and linearity of the poset implies that the interval $\left[\hat{0}, y_{j}\right]$ must be a chain, and since $x_{i}, x_{j} \in\left[\hat{0}, y_{j}\right], x_{i}$ and $x_{j}$ must be comparable. There are several possibilities:

1. $x_{j}<x_{i}<y_{i}<y_{j}$

2. $x_{i}<x_{j}<y_{i}<y_{j}$

3. $x_{i}<y_{i}<x_{j}<y_{j}$

In all three possibilities,

$$
\chi\left(x_{i}<y_{j}<y_{i}\right)+\chi\left(x_{j}<y_{i}<y_{j}\right)-\chi\left(x_{j}<x_{i}<y_{j}\right)-\chi\left(x_{i}<x_{j}<y_{i}\right)=0,
$$

and at the same time

$$
\chi\left(y_{i}<y_{j}\right)+\chi\left(y_{j}<y_{i}\right)-\chi\left(x_{i}<x_{j}\right)-\chi\left(x_{j}<x_{i}\right)=0 .
$$

On the other hand, if $y_{i}$ and $y_{j}$ are incomparable, then we have one of: 
1. $x_{j}<x_{i}<y_{i}, y_{j}$

2. $x_{i}<x_{j}<y_{i}, y_{j}$

3. $x_{i}<y_{i}, x_{j}<y_{j}, x_{i}$ and $x_{j}$ are incomparable.

Now in the first two cases

$$
\chi\left(x_{i}<y_{j}<y_{i}\right)+\chi\left(x_{j}<y_{i}<y_{j}\right)-\chi\left(x_{j}<x_{i}<y_{j}\right)-\chi\left(x_{i}<x_{j}<y_{i}\right)=-1,
$$

with

$$
\chi\left(y_{i}<y_{j}\right)+\chi\left(y_{j}<y_{i}\right)-\chi\left(x_{i}<x_{j}\right)-\chi\left(x_{j}<x_{i}\right)=-1
$$

too. In the last remaining case both expressions are zero.

Hence, the expression for the Laplacian above can be rewritten in the following form:

$$
L(\zeta)=(w(\zeta)+\Delta(\zeta)) \zeta+\sum_{y_{i}<P y_{j}} \zeta_{i, j}-\sum_{x_{i}<P} \zeta_{j}
$$

In other words, the meaning of the theorem above is that the Laplacian only transposes comparable labels of the element $z_{x_{1}, y_{1}} \wedge z_{x_{2}, y_{2}} \wedge \cdots \wedge z_{x_{k}, y_{k}}$, without introducing any new indices. This is the key observation for next section.

Lemma 10 Let $\zeta=z_{x_{1}, y_{1}} \wedge \cdots \wedge z_{x_{n}, y_{n}}$, and let $\zeta_{\sigma}=z_{x_{1}, y_{\sigma(1)}} \wedge z_{x_{2}, y_{\sigma(2)}} \wedge \cdots \wedge z_{x_{n}, y_{\sigma(n)}}$. If $\zeta_{\sigma} \neq 0$, i.e., if $x_{i}<_{P} y_{\sigma(i)}$ for all $i$, then

1. $w$ does not depend on $\sigma$, i.e.,

$$
w(\zeta)=w\left(\zeta_{\sigma}\right)
$$

2. $\Delta$ does not depend on $\sigma$, i.e.,

$$
\Delta(\zeta)=\Delta\left(\zeta_{\sigma}\right)
$$

This lemma actually proves that $w$ and $\Delta$ are dependent only on the choice of the (multi-)sets $X=\left\{x_{1}, x_{2}, \ldots, x_{k}\right\}, Y=\left\{y_{1}, y_{2}, \ldots, y_{k}\right\}$ (and a poset $P$ ), and not on the specific pure wedge constructed from those sets.

Proof: First we will check the claim for $w$.

$$
w(\zeta)=\sum_{i}\left|\left(x_{i}, y_{i}\right)\right|=\sum_{i} h t\left(y_{i}\right)-h t\left(x_{i}\right)-1,
$$

where the $h t(v)$ is the size of the interval $[\hat{0}, v]$. The sum on the right does not depend on $\sigma$, so we can write $w(X, Y)$ instead of $w(\zeta)$.

Now we will check the claim for $\Delta$.

$$
\begin{aligned}
\Delta(\zeta) & =\sum_{i<j}\left(\delta_{x_{i}, y_{j}}+\delta_{x_{j}, y_{i}}\right) \\
& =\sum_{i}\left(\text { multiplicity of } x_{i} \text { in the set } Y\right) \\
& =\sum_{j}\left(\text { multiplicity of } y_{j} \text { in the set } X\right) \\
& =\sum_{i, j} \delta_{x_{i}, y_{j}}
\end{aligned}
$$


which also does not depend on $\sigma$. Thus we can write $\Delta(X, Y)$ instead of $\Delta(\zeta)$ too.

We will use both notations, depending whether we want to stress $\zeta$ or the sets $(X, Y)$. Note that while $\Delta$ is completely determined by the sets $(X, Y), w$ also depends on the poset $P$ globally, i.e., it counts the sizes of intervals $\left(x_{i}, y_{i}\right)$ not relative to the sets $X$ and $Y$, but with respect to the whole poset $P$.

The simplicity of this formula is in the way the elements to which we are restricting the Laplacian, are obtained one from another, by simply transposing the labels. In general, this example shows that the Laplacian $L$ can be broken down into diagonal blocks, which are generated by a pure wedge $\zeta$, and all pure wedges obtained by permutations of the labels of $\zeta$. Furthermore, since $a \wedge b=-b \wedge a$, we can always keep the $x$-labels in order, i.e, we will always put the element $z_{x_{i}, *}$ at the $i^{\text {th }}$ position of the pure wedge.

\section{The eigenvalues of the Laplacian}

Let $\zeta=z_{x_{1}, y_{1}} \wedge z_{x_{2}, y_{2}} \wedge \cdots \wedge z_{x_{n}, y_{n}}$ be an element of the exterior algebra of the Lie algebra of $P$. In the last section we saw that the Laplacian acts on pure wedges of Lie algebra elements $z_{x_{1}, y_{1}} \wedge z_{x_{2}, y_{2}} \wedge \cdots \wedge z_{x_{n}, y_{n}}$ by summing the action of switching pairs of comparable $x$ 's, and pairs of comparable $y$ 's among themselves (plus a scalar).

That fact gives us the opportunity to divide our Laplacian into diagonal blocks where each block corresponds to all possible permutations of the $x$ 's and $y$ 's for a fixed choice of the element $z_{x_{1}, y_{1}} \wedge z_{x_{2}, y_{2}} \wedge \cdots \wedge z_{x_{n}, y_{n}}$, i.e., for the fixed choice of the multisets $X=$ $\left\{x_{1}, x_{2}, \ldots, x_{n}\right\}$, and $Y=\left\{y_{1}, y_{2}, \ldots, y_{n}\right\}$. In other words each block represents the "action" of the Laplacian on the subspace of the $n^{\text {th }}$ exterior power of our Lie algebra spanned by the elements $\left\{z_{x_{1}, y_{\sigma(1)}} \wedge z_{x_{2}, y_{\sigma(2)}} \wedge \cdots \wedge z_{x_{n}, y_{\sigma(n)}}: \sigma \in S_{n}\right\}$. Here the element $z_{x_{1}, y_{\sigma(1)}} \wedge z_{x_{2}, y_{\sigma(2)}} \wedge$ $\cdots \wedge z_{x_{n}, y_{\sigma(n)}}$ is defined if and only if $x_{i}<_{P} y_{\sigma(i)}$ for every $i=1,2, \ldots, n$. Thus each block is of size $n$ !, if all the elements are defined, or less, if some of the elements are not defined which is the case in general. The size of the block depends on the structure of the poset, and in particular, it depends on the relations in the subposet of $P$ spanned by the sets $X$ and $Y$. More formally :

Definition 4.1 The L-block $V$ spanned by the (multi)-sets $(X, Y)_{P}$, subsets of a poset $P$, is the vector space with basis

$$
\left\{z_{x_{1}, y_{\sigma(1)}} \wedge z_{x_{2}, y_{\sigma(2)}} \wedge \cdots \wedge z_{x_{n}, y_{\sigma(n)}}: \sigma \in S_{n}\right\}
$$

where $n=|X|=|Y|, \sigma$ is a permutation in $S_{n}$, and the element

$$
z_{x_{1}, y_{\sigma(1)}} \wedge z_{x_{2}, y_{\sigma(2)}} \wedge \cdots \wedge z_{x_{n}, y_{\sigma(n)}}
$$

is zero unless $x_{i}<_{P} y_{\sigma(i)}$ for all $i=1, \ldots, n$.

If we want to stress the dependence of the L-block $V$ of the sets $X$ and $Y$ and the poset $P$, we write $V(X, Y)_{P}$.

The sets $X$ and $Y$ may be multisets since some of the $x$ 's or $y$ 's might appear more than once as a label. In that case the sizes $|X|$ and $|Y|$ are counting multiplicities as well. 
Using this division of the chain space into L-blocks, we can use the results of the previous section, and state the theorem:

Theorem 11 Let $L_{P}$ be the Lie algebra corresponding to a linear poset $P$, and let $C_{n}\left(L_{P}\right)$ be the $n^{\text {th }}$ chain space. Then

$$
C_{n}\left(L_{P}\right)=\bigoplus_{(X, Y)} V(X, Y)_{P}
$$

where the direct sum is over all possible choices of (multi)-sets $X$ and $Y$ of equal cardinality, and each summand $V(X, Y)_{P}$ is invariant under the action of the Laplacian.

Thus we can now concentrate on the action of the Laplacian on each of these blocks.

\subsection{Embedding of the L-block in $\mathbb{C} S_{n}$}

Write the multisets $X$ and $Y$ as $X=\cup_{i \in A_{1}}\left\{x_{i}\right\} \cup \ldots \cup_{i \in A_{l}}\left\{x_{i}\right\}$, and $Y=\cup_{j \in B_{1}}\left\{y_{j}\right\} \cup$ $\ldots \cup_{j \in B_{m}}\left\{y_{j}\right\}$, where the $A_{i}$ 's contain the sets of indices of equal $x$ 's, and $B_{i}$ 's contain the sets of indices of equal $y$ 's.

For example, if $X=\left\{x_{1}, x_{2}, x_{3}, x_{4}, x_{5}\right\}$, where $x_{1}=x_{2}, x_{3}=x_{4}$, then $A_{1}=\{1,2\}$, $A_{2}=\{3,4\}$ and $A_{3}=\{5\}$.

Switching two of the $x$ 's will displace $x_{i}$ from its original position. To take into account the fact that we have to bring it back (by the choice of our basis) into the $i^{\text {th }}$ place, we need a minus sign.

Let

$$
\Pi_{x}=\sum_{\sigma_{1} \in \operatorname{Sym}\left(A_{1}\right), \sigma_{2} \in \operatorname{Sym}\left(A_{2}\right) \ldots}\left(\prod_{i} \operatorname{sgn}\left(\sigma_{i}\right)\right) \sigma_{1} \sigma_{2} \cdots \sigma_{l}
$$

and

$$
\Pi_{y}=\sum_{\sigma_{1} \in \operatorname{Sym}\left(B_{1}\right), \sigma_{2} \in \operatorname{Sym}\left(B_{2}\right) \ldots} \sigma_{1} \sigma_{2} \cdots \sigma_{m} .
$$

Then the L-block $V$ can be identified with a subspace of $\Pi_{x} \mathbb{C} S_{n} \Pi_{y}$. So $\Pi_{y}$ symmetrizes over equal $y$ 's and $\Pi_{x}$ anti-symmetrizes over equal $x$ 's. In other words, $\Pi_{x}$ permutes the positions, while $\Pi_{y}$ permutes indices.

\subsection{The Laplacian $L_{Y}$}

Let $X=\left\{x_{1}, \ldots, x_{n}\right\}$ and $Y=\left\{y_{1}, \ldots, y_{n}\right\}$ be two fixed (multi-)sets of vertices of the poset $P$, and consider the restriction of the Laplacian $L$ to L-block $V(X, Y)$.

To simplify the notation, we will write the Laplacian $L$ as:

$$
L(\zeta)=\left((w(X, Y)+\Delta(X, Y)) I d+\sum_{x_{i}<_{P} x_{j}}\left(x_{i}, x_{j}\right)+\sum_{y_{i}<_{P} y_{j}}\left(y_{i}, y_{j}\right)\right) \cdot \zeta
$$

where the "action" of $\left(y_{i}, y_{j}\right)$ or $\left(x_{i}, x_{j}\right)$ on $z_{x_{1}, y_{1}} \wedge z_{x_{2}, y_{2}} \wedge \cdots \wedge z_{x_{n}, y_{n}}$ means switching the corresponding pairs of $y$ 's, or $x$ 's. 
To simplify our examination, we will split it into these three parts:

$$
L=L_{D}+L_{X}+L_{Y},
$$

where

- $L_{D}$ is the scalar matrix, $L_{D}=w(X, Y)+\Delta(X, Y)$

- $L_{X}$ is the "action" of the Laplacian on the set of the $x$ 's, i.e.

$$
L_{X}=\sum_{x_{i}<P x_{j}}\left(x_{i}, x_{j}\right)
$$

- $L_{Y}$ is the "action" of the Laplacian on the set of the $y$ 's

$$
L_{Y}=\sum_{y_{i}<P}\left(y_{j}, y_{j}\right)
$$

on our L-block $V(X, Y)$.

In the embedding of this L-block into $\mathbb{C} S_{n}$, the notation for the Laplacian $L_{Y}$ would be $L_{Y}=\sum_{i<j: y_{i}<P y_{j}}(i, j)$, where the actual multiplication is from the right. The proper notation for $L_{X}$ in the $\Pi_{x} \mathbb{C} S_{n} \Pi_{y}$ is $L_{X}=\sum_{i<j: x_{i}<{ }_{P} x_{j}}(i, j)$, but the multiplication in this case is from the left.

Lemma $12 L_{Y}$ and $\Pi_{y}$ commute, i.e.,

$$
L_{Y} \cdot \Pi_{y}=\Pi_{y} \cdot L_{Y}
$$

Proof: It is sufficient to prove that the Laplacian $L_{Y}$ commutes with every transposition of the form $(i, k)$, where $y_{i}=y_{k}$, because every permutation in $\Pi_{y}$ can be written as a product of those permutations. So, let $y_{i}=y_{k}$. That means that $\Pi_{y}$ has transposition $(i, k)$ as one of its summands. Let $y_{j} \in Y$ be comparable to $y_{i}$ (thus it is comparable to $\left.y_{k}\right)$. In that case, the Laplacian $L_{Y}$ contains both transpositions, $(i, j)$, and $(k, j)$, i.e., $L_{Y}=\cdots+(i, j)+(k, j)+\cdots$.

But, $(i, k) \cdot(i, j)=(k, j) \cdot(i, k)$, which shows that $\Pi_{y} \cdot L_{Y}=L_{Y} \cdot \Pi_{y} \cdot$

Using exactly same argument we see that $L_{X}$ and $\Pi_{x}$ also commute.

We know from section 2 that $L_{D}$ is a scalar matrix on each block, and thus it commutes with $L_{X}$ and $L_{Y}$.

As for the $L_{X}$ and $L_{Y}$, we have the following Lemma:

\section{Lemma 13}

$$
L_{X} \cdot L_{Y} \cdot\left(z_{x_{1}, y_{1}} \wedge z_{x_{2}, y_{2}} \wedge \cdots \wedge z_{x_{n}, y_{n}}\right)=L_{Y} \cdot L_{X} \cdot\left(z_{x_{1}, y_{1}} \wedge z_{x_{2}, y_{2}} \wedge \cdots \wedge z_{x_{n}, y_{n}}\right)
$$


Proof:

The absence of certain relations in the poset may cause terms in the Laplacian to be missing. That is why this lemma is not obvious, and needs to be proved.

Let $\zeta=z_{x_{1}, y_{1}} \wedge z_{x_{2}, y_{2}} \wedge \cdots \wedge z_{x_{n}, y_{n}}$. Without loss of generality we can assume that all of the $x$ 's and all of the $y$ 's are distinct, because if they were not, we would just apply the same reasoning to each appearance of an observed element. Let $\left(x_{i}, x_{j}\right)$ be a transposition of the operator $L_{X}$, and let $\left(y_{k}, y_{l}\right)$ be a transposition of the operator $L_{Y}$. If all of the numbers $i, j, k, l$ are distinct, we have nothing to prove since it would not make any difference which transposition was applied first. On the other hand, if $i=k$ and $j=l$, again there is nothing to prove, since their combined action would amount to multiplying with -1 no matter in which order they are applied.

Therefore assume that $i \neq k$ but $j=l$, i.e., we have two transpositions, $\left(x_{i}, x_{j}\right)$ and $\left(y_{j}, y_{k}\right)$ in $L_{X}$ and $L_{Y}$ respectively, which overlap at one position. Without loss of generality assume that $n=3$. There are only three elements of the pure wedge, call them $z_{x_{1}, y_{1}} \wedge$ $z_{x_{2}, y_{2}} \wedge z_{x_{3}, y_{3}}$, i.e., $i=1, j=2, n=k=3$.

Let

$$
\begin{aligned}
\mathcal{A} & =\left(x_{1}, x_{2}\right) \cdot\left(y_{2}, y_{3}\right) \cdot\left(z_{x_{1}, y_{1}} \wedge z_{x_{2}, y_{2}} \wedge z_{x_{3}, y_{3}}\right) \\
& =\left(x_{1}, x_{2}\right) \cdot\left(z_{x_{1}, y_{1}} \wedge z_{x_{2}, y_{3}} \wedge z_{x_{3}, y_{2}}\right) \\
& =-\left(z_{x_{1}, y_{3}} \wedge z_{x_{2}, y_{1}} \wedge z_{x_{3}, y_{2}}\right)
\end{aligned}
$$

and

$$
\begin{aligned}
\mathcal{B} & =\left(y_{2}, y_{3}\right) \cdot\left(x_{1}, x_{2}\right) \cdot\left(z_{x_{1}, y_{1}} \wedge z_{x_{2}, y_{2}} \wedge z_{x_{3}, y_{3}}\right) \\
& =-\left(y_{2}, y_{3}\right) \cdot\left(z_{x_{1}, y_{2}} \wedge z_{x_{2}, y_{1}} \wedge z_{x_{3}, y_{3}}\right) \\
& =-\left(z_{x_{1}, y_{3}} \wedge z_{x_{2}, y_{1}} \wedge z_{x_{3}, y_{2}}\right) .
\end{aligned}
$$

Thus

$$
L_{X} \cdot L_{Y} \cdot\left(z_{x_{1}, y_{1}} \wedge z_{x_{2}, y_{2}} \wedge z_{x_{3}, y_{3}}\right)=L_{Y} \cdot L_{X} \cdot\left(z_{x_{1}, y_{1}} \wedge z_{x_{2}, y_{2}} \wedge z_{x_{3}, y_{3}}\right),
$$

whenever all of the relations used above are present, i.e., whenever every $x_{i}$ is beneath each $y_{j}$. That can be explained by the fact that $L_{X}$ is acting on the $x$-indices and $L_{Y}$ is acting on the $y$-indices.

The question remains whether the answer would be the same if some of the relations needed above were missing, and only one of the expressions above gets annulled. The final expressions in both $\mathcal{A}$ and $\mathcal{B}$ are 0 unless:

$$
x_{1}<y_{3}, \quad x_{2}<y_{1}, \quad x_{3}<y_{2} .
$$

Suppose (without loss of generality) that $\mathcal{B}$ above survives the procedure, i.e., we have the relation $x_{1}<y_{2}$. On the other hand if $\mathcal{A}$ is annulled in the middle step, the only possible conflict left is $x_{2} \nless y_{3}$. We have that $y_{2}$ and $y_{3}$ are comparable, otherwise the transposition $\left(y_{2}, y_{3}\right)$ wouldn't be a summand of $L_{Y}$. If $y_{2}<y_{3}$, then $x_{2}<y_{2}<y_{3}$, which is contrary to 
the just stated assumption. Thus, we must have $y_{3}<y_{2}$. Also $x_{1}<y_{3}$ by our assumption above, and $\left(x_{1}, x_{2}\right)$ is a transposition in $L_{X}$, so they must also be comparable. By the same argument as above, $x_{2}$ must be larger than $x_{1}$. Hence in the interval $\left(x_{1}, y_{2}\right)$ there are two elements $x_{2}$ and $y_{3}$. Since the poset is linear - those two elements must be comparable, and since we assumed that $x_{2} \nless y_{3}$, it must be that $x_{2}>y_{3}$.

All together, the relations are:

$$
\begin{aligned}
& y_{2} \\
& y_{1}
\end{aligned}>x_{2}>y_{3}>\begin{aligned}
& x_{3} \\
& x_{1}
\end{aligned}
$$

So in this case we have $y_{1}>y_{3}$, and $x_{2}>x_{3}$. Let

$$
\begin{aligned}
\mathcal{C} & =\left(x_{2}, x_{3}\right) \cdot\left(y_{1}, y_{3}\right) \cdot\left(z_{x_{1}, y_{1}} \wedge z_{x_{2}, y_{2}} \wedge z_{x_{3}, y_{3}}\right) \\
& =\left(x_{2}, x_{3}\right) \cdot\left(z_{x_{1}, y_{3}} \wedge z_{x_{2}, y_{2}} \wedge z_{x_{3}, y_{1}}\right) \\
& =-\left(z_{x_{1}, y_{3}} \wedge z_{x_{2}, y_{1}} \wedge z_{x_{3}, y_{2}}\right)
\end{aligned}
$$

and

$$
\begin{aligned}
\mathcal{D} & =\left(y_{1}, y_{3}\right) \cdot\left(x_{2}, x_{3}\right) \cdot\left(z_{x_{1}, y_{1}} \wedge z_{x_{2}, y_{2}} \wedge z_{x_{3}, y_{3}}\right) \\
& =-\left(y_{1}, y_{3}\right) \cdot\left(z_{x_{1}, y_{1}} \wedge z_{x_{2}, y_{3}} \wedge z_{x_{3}, y_{2}}\right) \\
& =0
\end{aligned}
$$

since $x_{2}>y_{3}$.

The expressions $\mathcal{A}$ and $\mathcal{C}$ are two summands of the product $L_{X} L_{Y}$, while $\mathcal{B}$ and $\mathcal{D}$ are two summands of the product $L_{Y} L_{X}$. As we can see, $\mathcal{A}+\mathcal{C}=\mathcal{B}+\mathcal{D}$. Thus $L_{X} \cdot L_{Y}=L_{Y} \cdot L_{X}$.

In view of Lemma $13, L_{X}, L_{Y}$ and $L_{D}$ are commuting linear transformations. So, to analyze the spectrum of their sum, we can compute the eigenvalues and eigenspaces of each separately. We will begin with $L_{Y}$.

\subsection{A poset tableau of type $(X, Y)_{P}$}

Definition 4.2 The diagram of the L-block, $P[X, Y]$, spanned by the sets $(X, Y)_{P}$, is the Hasse diagram of the subposet $X \cup Y$ with order inherited from the poset $P$. Furthermore every vertex of $P$, which is in the intersection $X \cap Y$ is split into two nodes, with the $x$-node above the $y$-node.

Definition 4.3 Given a node $v$ in $P[X, Y]$ define the repetition number of $v, k(v)$, to be the number of times that $v$ appears in the multiset $X$ if $v$ is an $x$-node of $P[X, Y]$, or the multiset $Y$ if $v$ is a $y$-node of $P[X, Y]$.

Let $C(v)$ be the set of covers of node $v$ in $P[X, Y]$. If $v$ is a maximal node, than $C(v)=\emptyset$. 
Definition $4.4 \mathrm{~A}$ poset tableau of type $(X, Y)_{P}$ (or just of type $(X, Y)$ ) is any labeling $\Lambda$ of the diagram, $P[X, Y]$, of the $L$-block $V$ spanned by $(X, Y)$, where the labels are partitions $\Lambda(v)$, such that $\Lambda(v)$ is a partition of the number $\sum_{w \geq v} \epsilon(w) k(w)$, where

$$
\epsilon(w)= \begin{cases}+1 & \text { if } w \text { is a } y \text {-node } \\ -1 & \text { if } w \text { is an } x \text {-node }\end{cases}
$$

Given a poset tableau $\Lambda$ we will define the multiplicity of $\Lambda, m(\Lambda)$, and the eigenvalues of $\Lambda, e(\Lambda)$.

Definition 4.5 - Let $v$ be a y-node of the diagram $P[X, Y]$, labeled with the partition $\Lambda(v)$ and with repetition number $k(v)$. Let $C(v)=\left\{v_{1}, v_{2}, \ldots, v_{l}\right\}$ be the set of covers of $v$. Let $\lambda_{i}$ denote $\Lambda\left(v_{i}\right)$, and let $k_{i}$ denote the repetition numbers, $k\left(v_{i}\right)$. The multiplicity of $\Lambda$ at $v$ is defined to be

$$
m_{v}(\Lambda)=c_{\lambda_{1}, \ldots, \lambda_{l}, k(v)}^{\Lambda(v)}
$$

- Let $v$ be an $x$-node of the diagram $P[X, Y]$, labeled with the partition $\Lambda(v)$ and with repetition number $k(v)$. Let $C(v)=\left\{v_{1}, v_{2}, \ldots, v_{l}\right\}$ be the set of covers of $v$. Let $\lambda_{i}$ denote $\Lambda\left(v_{i}\right)$, and let $k_{i}$ denote the repetition numbers, $k\left(v_{i}\right)$. The multiplicity of $\Lambda$ at $v$ is defined to be

$$
m_{v}(\Lambda)=\sum_{\mu} c_{\lambda_{1}, \ldots, \lambda_{l}}^{\mu} c_{\Lambda(v), 1^{k(v)}}^{\mu}
$$

If the multiplicity $m_{v}(\Lambda)=0$ then we know that that particular labeling is not valid.

Now, we will define the $y$-eigenvalues for each $y$-node $v$ of the diagram $P[X, Y]$. We want to have as many $y$-eigenvalues as the value of multiplicity. From the representation theory of the symmetric group, we know that

$$
c_{\lambda_{1}, \ldots, \lambda_{l}, k(v)}^{\Lambda(v)}=\sum_{\Lambda(v) / \mu=k(v) \text {-horizontal strip }} c_{\lambda_{1}, \ldots, \lambda_{l}}^{\mu} .
$$

The node-eigenvalue, $e_{v}(\Lambda)$, for each node $v$, is the set of the sums of the content over all squares in $\Lambda(v) / \mu$ for all possible $\mu$ for which $\Lambda(v) / \mu$ is a $k(v)$-horizontal strip minus the binomial coefficient $\left(\begin{array}{c}k(v) \\ 2\end{array}\right)$.

Recall that the content of a square is given by $c(i, k)=k-i$ if the square is at position $(i, k)$ in a partition ( $i^{\text {th }}$ row and $k^{\text {th }}$ column).

This gives $m_{v}(\Lambda)$ eigenvalues at each $y$-node $v$. We now define $y$-eigenvalue of $\Lambda$, $e_{y}(\Lambda)$, to be the set of numbers obtained by taking a sum of one element of $e_{v}(\Lambda)$ for each $y$-node $v$. So $\left|e_{y}(\Lambda)\right|=\prod_{y \text {-nodes } v} m_{v}(\Lambda)$.

\subsection{Example}

Let the poset $P=\{1,2,3,4,5\}$ with the relations $1<_{P} 2,2<_{P} 3,3<_{P} 4$ and $4<_{P} 5$. The Hasse diagram of this poset is given in figure 1 . 


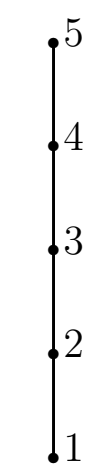

Figure 1: Example: poset $P$

Let $X$ and $Y$ be the sets $X=\{1,2,3\}$ and $Y=\{4,4,5\}$. So the node 4, is a node with non-trivial repetition number $k(4)=2$. The L-block $V$ is spanned by the following pure wedges:

$$
\begin{aligned}
\zeta & =z_{1,4} \wedge z_{2,4} \wedge z_{3,5} \\
\tau & =z_{1,4} \wedge z_{2,5} \wedge z_{3,4} \\
\eta & =z_{1,5} \wedge z_{2,4} \wedge z_{3,4}
\end{aligned}
$$

Thus the L-block $V$ is 3-dimensional. We calculate the Laplacian $L_{Y}$ on these three elements. Note that the Laplacian $L_{Y}$ is in fact $L_{Y}=(4,5)$, since those are the only two comparable $y$ 's.

$$
\begin{aligned}
& L(\zeta)=\tau+\eta \\
& L(\tau)=\zeta+\eta \\
& L(\eta)=\zeta+\tau
\end{aligned}
$$

The matrix representation of $L_{Y}$ with respect to the basis $\langle\zeta, \tau, \eta\rangle$ is thus

$$
L_{Y}=\left(\begin{array}{ccc}
0 & 1 & 1 \\
1 & 0 & 1 \\
1 & 1 & 0
\end{array}\right)
$$

So the eigenvalues of the Laplacian are $-1,-1,+2$.

Now we will evaluate the $y$-eigenvalue for each of the poset tableaux for this L-block. The only nontrivial node is node 4 . Thus, the $y$-eigenvalue, $e_{y}(\Lambda)$, is the node-eigenvalue, $e_{4}(\Lambda)=c(1,2)+c(1,3)-\left(\begin{array}{l}2 \\ 2\end{array}\right)$. The result is given in figure 2 .

Note that the $y$-eigenvalues of this labeling give exactly the same numbers as the eigenvalues of the Laplacian $L_{Y}$. In the next section we will show that this is not coincidental. 

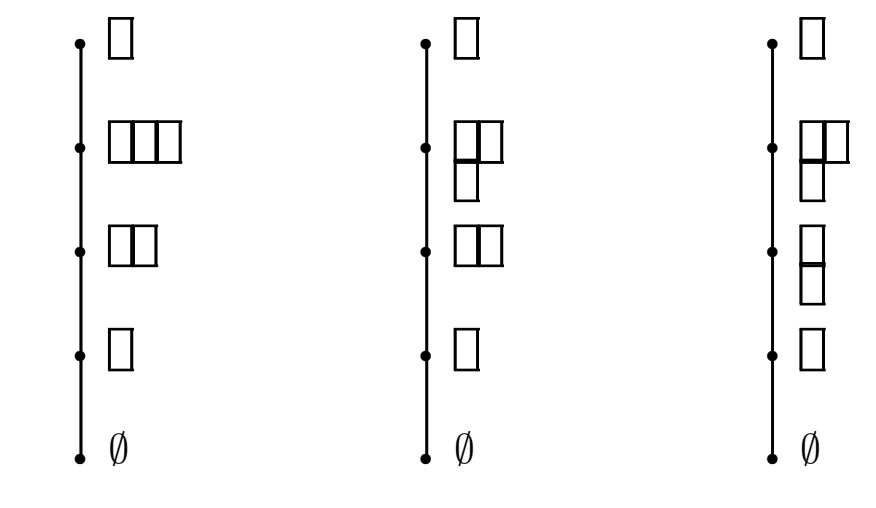

$\overline{e_{y}(\Lambda)}=2$

$\overline{e_{y}(\Lambda)}=-1$

$\overline{e_{y}(\Lambda)}=-1$

Figure 2: Example: the $y$-eigenvalues

\section{Centerpiece Theorem for $L_{Y}$}

Theorem 14 ( $L_{Y}$-Centerpiece) Let $P$ be a linear poset with a minimum element, $\hat{0}$. Let $X$ and $Y$ be two (multi-)sets, subsets of $P$. For every labeling $\Lambda$ of positive multiplicity, each element in $e_{y}(\Lambda)$ is an eigenvalue of $L_{Y}$ with multiplicity $\prod_{x \text {-nodes } v} m_{v}(\Lambda)$.

Proof:

The proof of this theorem will be by induction on the sizes of the (multi)-sets $X$ and $Y$. So let $n=|X|=|Y|$ (counting multiplicities).

If $n=1$ - there is nothing to prove as the Laplacian $L_{Y}$ has no pairs to switch, and the only $y$-node is the maximal element for the diagram of the L-block. The Laplacian $L_{Y}$ is the one-by-one zero matrix and the eigenvalue of this unique pair is zero.

Suppose $n=2$. There are several different possible combinations of relations between sets $X=\left\{x_{1}, x_{2}\right\}$ and $Y=\left\{y_{1}, y_{2}\right\}$.

- The most obvious one is $x_{1}<x_{2}<y_{1}<y_{2}$. In that case the Laplacian $L_{Y}=\left(y_{1}, y_{2}\right)$, and the two possible elements are $\zeta_{1}=z_{x_{1}, y_{1}} \wedge z_{x_{2}, y_{2}}, \zeta_{2}=z_{x_{1}, y_{2}} \wedge z_{x_{2}, y_{1}}$. The Laplacian $L_{Y}$ has the following matrix representation with respect to the basis $\left\langle\zeta_{1}, \zeta_{2}\right\rangle$ :

$$
L_{Y}=\left(\begin{array}{ll}
0 & 1 \\
1 & 0
\end{array}\right)
$$

The eigenvalues of $L_{Y}$ are +1 and -1 . The eigenvalue of the poset tableau of type $(X, Y)_{P}$ is given in figure 3 . It also gives values +1 and -1 , so the claim of the theorem holds. 


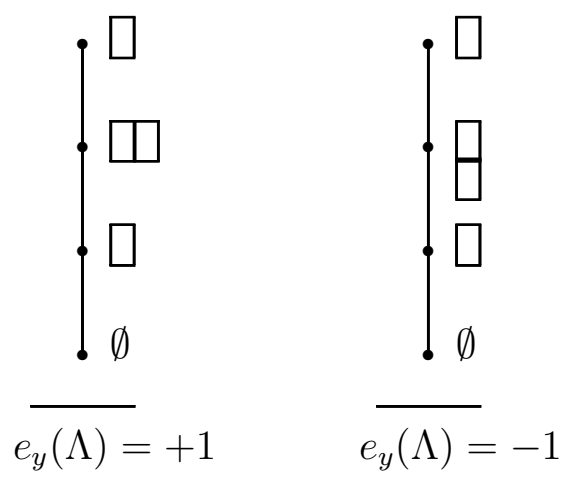

Figure 3: poset tableaux

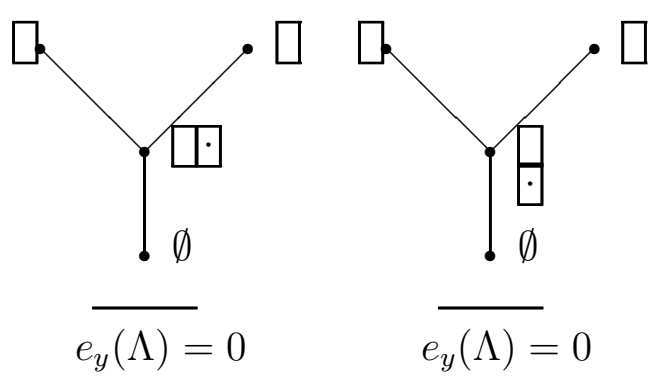

Figure 4: poset tableaux

- The second case is when

$$
x_{1}<x_{2}<\begin{aligned}
& y_{1} \\
& y_{2}
\end{aligned}
$$

In that case the Laplacian $L_{Y}$ does nothing (since $y_{1}$ and $y_{2}$ are not comparable), thus the eigenvalues of $L_{Y}$ are 0 . The dimension of the L-block spanned by $(X, Y)$ is two. The eigenvalues of the poset tableaux give the same values (figure 4), where the ". in a box denotes which square was deleted in that step.

Thus in this case the theorem checks too.

- $x_{1}<y_{1}<x_{2}<y_{2}$ or equivalently (for our purpose) $x_{1}<y_{1}=x_{2}<y_{2}$.

There is only one poset tableau spanned by these sets $X$ and $Y$, namely the one shown on the figure 5 .

The $y$-eigenvalue for the poset tableau is zero in both cases.

The Laplacian $L_{Y}$ can not switch the $y$ 's, since that would produce the element $z_{x_{2}, y_{1}}$ which doesn't exist. So the Laplacian $L_{Y}$ also acts as zero. 


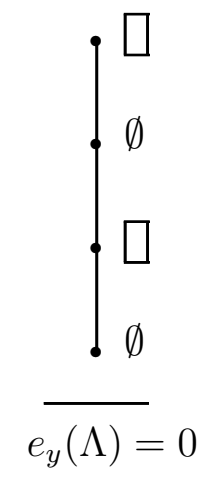

Figure 5: poset tableau

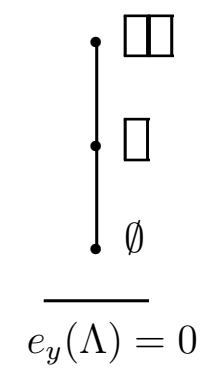

Figure 6: poset tableau

- $x_{1}<x_{2}<y_{1}=y_{2}$.

There is only one poset tableau spanned by these sets $X$ and $Y$, namely the one shown on the figure 6 .

The $y$-eigenvalue is again zero (contents of the partition (2) minus the binomial coefficient $\left(\begin{array}{l}2 \\ 2\end{array}\right)$ ). The Laplacian $L_{Y}$ doesn't have two distinct $y$ 's to switch, thus, it is zero.

- $x$ 's are the same.

$$
x_{1}=x_{2}<\begin{aligned}
& y_{1} \\
& y_{2}
\end{aligned}
$$

There is only one poset tableau spanned by these sets $X$ and $Y$, namely the one shown on the figure 7 .

The $y$-eigenvalue is zero. The Laplacian $L_{Y}$ has no comparable $y$ 's to switch - thus $L_{Y}=0$.

- $x_{1}=x_{2}<y_{1}<y_{2}$. There is only one poset tableau spanned by these sets $X$ and $Y$, namely the one shown on the figure 8 .

The $y$-eigenvalue is equal to 1 . The Laplacian $L_{Y}$ can switch $y_{1}$ and $y_{2}$ but the result would be the same element, since the $x$ 's are indistinguishable. Thus the Laplacian 


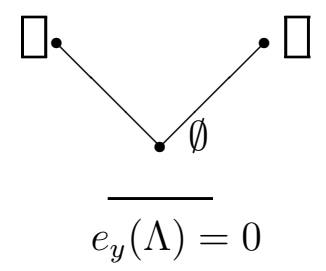

Figure 7: poset tableau

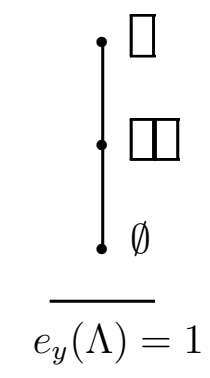

Figure 8: poset tableau

$L_{Y}=I d$, with the eigenvalue 1.

- In the trivial case when the $x$ 's are not comparable the $y$ 's are not comparable because of linearity and the existence of a minimal element. So we have

$$
\begin{aligned}
& x_{1}<y_{1} \\
& x_{2}<y_{2}
\end{aligned} .
$$

The poset tableau again gives zero as the $y$-eigenvalue, and since the $y$ 's are not comparable, the Laplacian $L_{Y}$ is also zero.

So the theorem holds for the case $n=2$.

Now, we will treat the general case $n>2$.

- Label the $y$-nodes of the diagram of the L-block using the depth-first algorithm:

1. Start with a leftmost minimal $y$-element $v$.

2. If $v$ is not the maximal unlabeled $y$-node go to the leftmost unlabeled cover of $v$, and repeat this step. Otherwise label $v$ with next available number from the set $\{1,2, \ldots,|Y|\}$.

From this labeling we see that, $y_{i}>y_{j} \Rightarrow i<j$.

- Let $P\left[X_{1}, Y_{1}\right], P\left[X_{2}, Y_{2}\right], \ldots, P\left[X_{c}, Y_{c}\right]$ be the connected components of $P[X, Y]$. In that case the L-block $V$ is the tensor product of the L-blocks of the $P\left[X_{i}, Y_{i}\right]$. The 
Laplacian $L_{Y}$ switches only comparable $y$ 's, and two $y$ 's from different connected components are not comparable. Thus

$$
L_{Y}\left(v_{1} \otimes \cdots \otimes v_{c}\right)=\sum_{i=1}^{c} v_{1} \otimes \cdots \otimes\left(L_{Y_{i}} v_{i}\right) \otimes \cdots \otimes v_{c} .
$$

So if $v_{1}, \ldots, v_{c}$ are eigenvectors of $L_{Y}$ with the eigenvalues $e_{1}, \ldots, e_{c}$, then $v_{1} \otimes \cdots \otimes v_{c}$ is an eigenvector with eigenvalue $e_{1}+\cdots+e_{c}$. Thus, by induction, we can label each of the components of $P[X, Y]$ to get the eigenvalues of $L_{Y}$ on the total L-block $V$.

- Suppose that $P[X, Y]$ is connected. In that case, there must be a minimal element in $P[X, Y]$, which must be an $x$-node.

Call the minimal element $x_{n}$. Then define $x_{n-1}, x_{n-2}, \ldots, x_{a}$ by:

1. $x_{a}>x_{a+1}>\cdots>x_{n}$, where all $>$ are covering relations.

2. Either

Case 1: There is more than one element covering $x_{a}$.

Case 2: $x_{a}$ has unique cover in $P[X, Y]$ but it is a $y$-element.

Let $B=\left\{x_{a}, \ldots, x_{n}\right\}$, and let $G=\operatorname{Sym}(B)$.

Lemma 15 Let $\sigma \in G$, and let $\zeta=z_{x_{i_{1}}, y_{1}} \wedge z_{x_{i_{2}}, y_{2}} \wedge \cdots \wedge z_{x_{i_{n}}, y_{n}}$ be non-zero. Then

$$
\zeta^{\sigma}=z_{\sigma\left(x_{i_{1}}\right), y_{1}} \wedge z_{\sigma\left(x_{i_{2}}\right), y_{2}} \wedge \cdots \wedge z_{\sigma\left(x_{i_{n}}\right), y_{n}}
$$

is also non-zero.

Proof: It is sufficient to prove the lemma for the transposition $\left(x_{i_{k}}, x_{i_{l}}\right) \in G$.

$$
\zeta^{\left(x_{i_{k}}, x_{i_{l}}\right)}=z_{x_{i_{1}}, y_{1}} \wedge \cdots \wedge z_{x_{i_{l}}, y_{l}} \wedge \cdots \wedge z_{x_{i_{k}}, y_{k}} \wedge \cdots \wedge z_{x_{i_{n}}, y_{n}}
$$

Now, since $\left(x_{i_{k}}, x_{i_{l}}\right) \in B$, i.e., $x_{i_{k}}, x_{i_{l}}$ are both less or equal to $x_{a}$, which is below all of the $y$ 's - the lemma is clear.

Lemma 16 This action of $G$ commutes with $L_{Y}$.

Proof: Let $\zeta=z_{x_{i_{1}}, y_{1}} \wedge z_{x_{i_{2}}, y_{2}} \wedge \cdots \wedge z_{x_{i_{n}}, y_{n}}$ be in our L-block, $V$, let $y_{k}<_{P} y_{l}$ and let $\sigma \in G$. Then

$$
\sigma \cdot\left(y_{k}, y_{l}\right) \cdot \zeta=z_{\sigma\left(x_{i_{1}}\right), y_{1}} \wedge \cdots \wedge z_{\sigma\left(x_{i_{k}}\right), y_{l}} \wedge \cdots \wedge z_{\sigma\left(x_{i_{l}}\right), y_{k}} \wedge \cdots \wedge z_{\sigma\left(x_{i_{n}}\right), y_{n}}
$$

and

$$
\left(y_{k}, y_{l}\right) \cdot \sigma \cdot \zeta=z_{\sigma\left(x_{i_{1}}\right), y_{1}} \wedge \cdots \wedge z_{\sigma\left(x_{i_{k}}\right), y_{l}} \wedge \cdots \wedge z_{\sigma\left(x_{i_{l}}\right), y_{k}} \wedge \cdots \wedge z_{\sigma\left(x_{i_{n}}\right), y_{n}}
$$




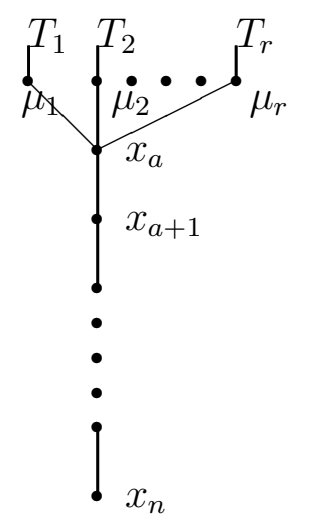

Figure 9: Case 1

So they are equal unless one of the expressions above is zero, and the other is not. The only way for that to happen is in the middle step, i.e., either $\sigma \cdot \zeta=0$ or $\left(y_{k}, y_{l}\right) \cdot \zeta=0$. But we assumed the $\zeta \neq 0$, and by our lemma above $\sigma \cdot \zeta \neq 0$. So the only possible conflict is $\left(y_{k}, y_{l}\right) \cdot \zeta=0$, and $\left(y_{k}, y_{l}\right) \cdot \sigma \cdot \zeta \neq 0$. But since $\sigma \in G$, it only moves elements of $B$ which are allowed to be paired with any $y_{k}$

Let $C\left(x_{a}\right)=\left\{v_{1}, v_{2}, \ldots, v_{l}\right\}$ be the set of covers of $x_{a}$. We will prove the following generalization of the Centerpiece Theorem:

Theorem 17 Let $\Lambda$ be a poset tableau of positive multiplicity, and let $\lambda_{i}$ be the label of $v_{i}$ in $\Lambda$. Let $\lambda$ be a partition such that

$$
c_{\lambda_{1}, \ldots, \lambda_{l}}^{\lambda} c_{\Lambda\left(x_{a}\right), 1^{k\left(x_{a}\right)}}^{\lambda} \neq 0
$$

Then the occurrences of $G$-irreducibles $S^{\lambda}$ in $V$ can be indexed by such poset tableaux $\Lambda$, and the Laplacian $L_{Y}$ acts on $S^{\lambda}$ as one of the scalars in $e_{Y}(\Lambda)$.

\subsubsection{Case 1}

Suppose there are two or more subtrees above the node $x_{a}$, in our poset $P$ (as in figure 9). Label the subtrees above the $x_{a}$ by $T_{1}, T_{2}, \ldots, T_{r}$. Let $Y=Y_{1} \cup Y_{2} \cup \cdots \cup Y_{r}$ $\left(Y_{i} \subset T_{i}\right)$, where $Y_{i} \cap Y_{j}=\emptyset$. Let $k_{i}=\left|Y_{i}\right|$, and let

$$
Y_{i}=\left\{y_{k_{1}+k_{2}+\cdots+k_{i-1}+1}, \ldots, y_{k_{1}+\cdots+k_{i-1}+k_{i}}\right\} .
$$

Because of our labeling, we know that all relations between $y$ 's are contained within the sets $Y_{i}$, i.e., $y_{i}<_{P} y_{j}$ implies that both $y_{i}, y_{j}$ are in the same $Y_{k}$.

Let $b_{i}$ be the number of $y_{j}$ 's in $T_{i}$ minus the number of $x_{j}$ 's in $T_{i}$ (note that in general $T_{i}$ will have more $y_{j}$ 's than $x_{j}{ }^{\prime}$ 's). In other words, $b_{i}=\left|Y_{i}\right|-\left|X \cap T_{i}\right|$. 
Consider element $\zeta=z_{x_{i_{1}}, y_{1}} \wedge z_{x_{i_{2}}, y_{2}} \wedge \cdots \wedge z_{x_{i_{n}}, y_{n}}$. If we want $\zeta$ to be non-zero, there will be exactly $b_{i} x_{j}$ 's from $B$ in $\zeta$ paired up with the $y_{j}$ 's of $T_{i}$.

Split the L-block

$$
V=\oplus_{\left(S_{1}, S_{2}, \ldots, S_{r}\right)} V\left[S_{1}, S_{2}, \ldots, S_{r}\right],
$$

where $S_{1} \cup S_{2} \cup \cdots \cup S_{r}=B,\left|S_{i}\right|=b_{i}$, and $V\left[S_{1}, \ldots, S_{r}\right]$ is the span of all $\zeta$ with exactly the elements of $S_{i}$ paired with the $y$ 's from $T_{i}$.

Lemma 18 1. As a vector space

$$
V\left[S_{1}, \ldots, S_{r}\right] \cong V\left(X_{1} \cup S_{1}, Y_{1}\right) \otimes V\left(X_{2} \cup S_{2}, Y_{2}\right) \otimes \cdots \otimes V\left(X_{r} \cup S_{r}, Y_{r}\right),
$$

where $X_{i}$ and $Y_{i}$ are the multisets of the $x$ and $y$ elements of the subtree $T_{i}$.

2. With respect to the decomposition in 1., the Laplacian $L_{Y}$ acts as:

$$
L_{Y}\left(v_{1} \otimes \cdots \otimes v_{r}\right)=\sum_{i} v_{1} \otimes \cdots \otimes\left(L_{Y_{i}} v_{i}\right) \otimes \cdots \otimes v_{r} .
$$

Proof: Statement 1. is clear by the definition of $V\left[S_{1}, \ldots, S_{r}\right]$. To prove statement 2 ., we only need to recall that the Laplacian $L_{Y}$ switches comparable $y$ 's, and that $y$ 's in different subtrees can not be comparable because of linearity of the poset. $L_{Y}$ can switch only $y$ 's in the same subtree $T_{i}$.

Let $G_{i}=\operatorname{Sym}\left(S_{i}\right)$. Note that $G_{1} \times G_{2} \times \ldots \times G_{r}$ acts on $V\left[S_{1}, S_{2}, \ldots, S_{r}\right]$. Let $s_{i}$ be the minimal node of subtree $T_{i}$.

Now apply the induction hypothesis to L-blocks, $V_{i}=V\left(X_{i} \cup S_{i}, Y_{i}\right)$. According to our theorem this gives the decomposition of the L-block $V_{i}$ as $G_{i}$-module, and the eigenvalues of $L_{Y_{i}}$ are indexed by poset tableau of shape $\mu_{i} \vdash\left|S_{i}\right|$. Moreover each poset tableau of shape $\mu_{i}$ with eigenvalue $e_{i}$ represents a copy of the irreducible $S^{\mu_{i}}$ in the $e_{i}$-eigenspace.

Now, as a $G_{1} \times \ldots \times G_{r}$ module we know the eigenspaces of $L_{Y}$ are given by our labeling up to the points $s_{i}$ where the partitions $\mu_{i}$ come together at $x_{a}$.

Lemma 19 As a $G=\operatorname{Sym}(B)$-module, the space $V$ is

$$
V \cong \operatorname{ind}_{\left(\operatorname{Sym}\left(S_{1}^{0}\right) \times \cdots \times \operatorname{Sym}\left(S_{r}^{0}\right)\right)}^{\operatorname{Sym}(B)}\left(V\left[S_{1}^{0}, \ldots, S_{r}^{0}\right]\right),
$$

where $\left(S_{1}^{0}, \ldots, S_{r}^{0}\right)$ is any fixed ordered partition of $B$.

Proof: Choose $S_{i}^{0}=\left\{x_{a+b_{1}+\cdots+b_{i-1}}, x_{a+b_{1}+\cdots+b_{i-1}+1}, \ldots, x_{a+b_{1}+\cdots+b_{i}-1}\right\}$. Let $\mathcal{S} h$ denote the set of permutations $\sigma \in \operatorname{Sym}(B)$ such that $\sigma(u)<\sigma(v)$ whenever $u, v$ are in the same set $S_{i}^{0}$ for some $i$. There is $1-1$ correspondence between the $\sigma \in \mathcal{S} h$ and the sequences indexing the summands in the 15 , namely

$$
\sigma \leftrightarrow\left(S_{1}^{\sigma}, \ldots, S_{r}^{\sigma}\right)
$$


where $S_{i}^{\sigma}=\left\{\sigma(u): u \in S_{i}^{0}\right\}$. Also $\mathcal{S} h$ is a collection of coset representatives for $\operatorname{Sym}\left(S_{1}^{0}\right) \times \cdots \times \operatorname{Sym}\left(S_{r}^{0}\right)$ in $\operatorname{Sym}(B)$. Thus we have a natural vector space isomorphism between $V$ and

$$
V\left[S_{1}^{0}, \ldots, S_{r}^{0}\right] \otimes_{\left(\operatorname{Sym}\left(S_{1}^{0}\right) \times \cdots \times \operatorname{Sym}\left(S_{r}^{0}\right)\right)} \operatorname{Sym}(B) .
$$

It is straightforward to check that this isomorphism commutes with the action of $\operatorname{Sym}(B)$

Let $\Lambda_{i}$ be a poset tableau of type $\left(X_{i} \cup S_{i}, Y_{i}\right)$, where $\mu_{i} \vdash b_{i}$ is the label of the vertex $s_{i}$. By our inductive hypothesis the Laplacian $L_{Y}$ acts as a scalar on the irreducible $S^{\mu_{i}}$, i.e., $\left.L_{Y}\right|_{S^{\mu_{i}}}=e_{Y}\left(\Lambda_{i}\right)$. Applying Lemma 18 part 2., we have

$$
\begin{aligned}
L_{Y}\left(v_{1} \otimes \cdots \otimes v_{r}\right) & =\sum_{i} v_{1} \otimes \cdots \otimes\left(L_{Y_{i}} v_{i}\right) \otimes \cdots \otimes v_{r} \\
& =\sum_{i} v_{1} \otimes \cdots \otimes e_{Y}\left(\Lambda_{i}\right) v_{i} \otimes \cdots \otimes v_{r} \\
& =\left(\sum_{i} e_{Y}\left(\Lambda_{i}\right)\right)\left(v_{1} \otimes \cdots \otimes v_{r}\right)
\end{aligned}
$$

Now, we will use the fact $([16,17,24])$ that

$$
\left(S^{\mu_{1}} \otimes \cdots \otimes S^{\mu_{r}}\right) \uparrow_{G_{1} \times \cdots \times G_{r}}^{G}=\oplus_{\lambda \vdash|B|} c_{\mu_{1}, \mu_{2}, \ldots, \mu_{r}}^{\lambda} S^{\lambda} .
$$

Thus we have $c_{\mu_{1}, \mu_{2}, \ldots, \mu_{r}}^{\lambda}$ copies of the $G$-module $S^{\lambda}$ which explains why this is the multiplicity of the label $\lambda$ on node $x_{a}$ in our labeling.

- Now we have to decide what is the dimension of each eigenspace. But that is something we will have to do in the second case too - so we will do it for both cases at the end.

\subsubsection{Case 2}

Let $A=\left\{y_{n-k+1}, \ldots, y_{n}\right\}$ be the largest possible set so that

$$
x_{a} \leq_{P} y_{n} \leq_{P} y_{n-1} \leq_{P} \cdots \leq_{P} y_{n-k+1}
$$

and there are no $x_{i}$ 's with $y_{n} \leq_{P} x_{i}<y_{n-k+1}$. Call $A$ the "terminal $Y$-set of $V$ " (figure 10). Note that $|B| \geq|A|$. Split the L-block

$$
V=\oplus_{\left(a_{1}, \ldots, a_{k}\right)} V\left(a_{1}, \ldots, a_{k}\right)
$$

for $\left(a_{1}, \ldots a_{k}\right)$, a sequence of distinct elements of length $k$ from $B$, and the vector space $V\left(a_{1}, a_{2}, \ldots, a_{k}\right)$ represents the span of all $\zeta$ which are of the form

$$
\zeta=z_{x_{1}, y_{1}} \wedge \cdots \wedge z_{x_{n-k}, y_{n-k}} \wedge z_{a_{1}, y_{n-k+1}} \wedge \cdots \wedge z_{a_{k}, y_{n}}
$$




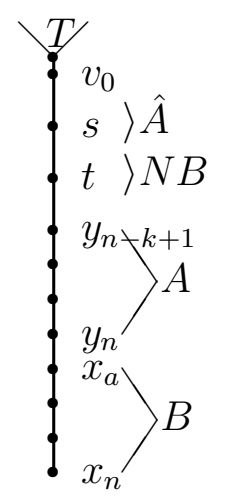

Figure 10: Case 2

For the moment assume that all of the elements $x_{a}, \ldots, x_{n}$ are distinct and all of $y_{n-k+1}, \ldots, y_{n}$ are distinct. Then we will look back at how we must modify the argument when some of the $x_{i}$ 's and $y_{j}$ 's are equal.

Fix the sequence $\left(a_{1}, \ldots, a_{k}\right)$, let $B^{\prime}=B \backslash\left\{a_{1}, \ldots, a_{k}\right\}$ and let $G^{\prime}$ be the subgroup $G^{\prime}=\operatorname{Sym}\left(B^{\prime}\right) \leq G$. Note that $G^{\prime}$ acts on $V\left(a_{1}, \ldots, a_{k}\right)$.

Lemma $20 V\left(a_{1}, \ldots, a_{k}\right)$ is isomorphic to the L-block $V_{0}$ given by the following sets: $X^{\prime}=X \backslash\left\{a_{1}, a_{2}, \ldots, a_{k}\right\}$ and $Y^{\prime}=Y \backslash\left\{y_{n-k+1}, \ldots, y_{n}\right\}$.

Proof: The isomorphism $\psi: V\left(a_{1}, \ldots, a_{k}\right) \rightarrow V_{0}$ is an obvious one

$$
\psi\left(z_{x_{1}, y_{1}} \wedge \cdots \wedge z_{x_{n-k}, y_{n-k}} \wedge z_{a_{1}, y_{n-k+1}} \wedge \cdots \wedge z_{a_{k}, y_{n}}\right)=z_{x_{1}, y_{1}} \wedge \cdots \wedge z_{x_{n-k}, y_{n-k}}
$$

It is clearly a bijective linear map.

- Now let's examine the L-block given by $X^{\prime}$ and $Y^{\prime}$. Let $\hat{A}$ be the terminal $Y$-set for $X^{\prime}, Y^{\prime}$. Let $\hat{B}=B^{\prime} \cup\left\{\right.$ new $x_{i}$ 's $\}$ be the $x_{i}$ 's below $\hat{A}$. Let $N B$ denote the set of those new $x_{i}$ 's. Let $\hat{G}=\operatorname{Sym}(\hat{B})$. Note that $G^{\prime} \leq \hat{G}$.

If $\hat{A}=\emptyset$ then there are no $y$ 's in the interval $\left(y_{n-k+1}, v_{0}\right]$. In that case the group $\hat{G}$ is the group described in the case 1., i.e., $\hat{G}=G_{1} \times G_{2} \times \cdots \times G_{r}$, where $G_{i}$ is acting on the subtree $T_{i}$ above $x_{a}$.

Now apply the induction hypothesis to $X^{\prime}, Y^{\prime}$. This gives the decomposition of the L-block as a $\hat{G}$-module. The theorem says that the irreducible summands are indexed by the $\left(X^{\prime}, Y^{\prime}\right)$ poset tableaux $\hat{\Lambda}$ of shape $\lambda^{\prime}$ (where $\lambda^{\prime} \vdash|\hat{B}|$ ), and "shape" means that the minimal element $s$ of $\hat{A}$ is labeled with $\lambda^{\prime}$. Also the theorem tells us that the $Y$-Laplacian $L_{Y}$ for $\left(X^{\prime}, Y^{\prime}\right)$ acts like the scalar $e_{Y}(\hat{\Lambda})$ on this copy of the irreducible $S^{\lambda^{\prime}}$. From this we can deduce by restriction from $\hat{G}$ to $G^{\prime}$ the following 
Lemma 21 As a $G^{\prime}$-module, the space $V\left(a_{1}, \ldots, a_{k}\right)$ decomposes as a sum over all $\left(X^{\prime}, Y^{\prime}\right)$ tableaux $\hat{\Lambda}$ of shape $\lambda^{\prime}$ of the module

$$
S^{\lambda^{\prime}} \downarrow_{G^{\prime}}^{\hat{G}}
$$

Moreover the Laplacian $L_{Y}$ for $\left(X^{\prime}, Y^{\prime}\right)$ acts like the scalar $e_{Y}(\hat{\Lambda})$ on this entire restriction.

Let $\mathcal{C h}\left(\lambda^{\prime}, \mu^{\prime}\right)$ be the set of chains $\lambda^{\prime} \geq \lambda_{1} \geq \cdots \geq \mu^{\prime}$ where the steps in the chain of the partitions are all of size 1 .

Now using the fact [16] that

$$
\left(S^{\lambda^{\prime}}\right) \downarrow_{G^{\prime}}^{\hat{G}}=\oplus_{\mu^{\prime} \vdash\left|B^{\prime}\right|} S^{\mu^{\prime}}\left|\mathcal{C h}\left(\lambda^{\prime}, \mu^{\prime}\right)\right|,
$$

we can rewrite this lemma to say that the sum is over all extensions of $\hat{\Lambda}$ to a labeling of the new $x_{i}$ 's of $S^{\mu^{\prime}}$ where the extension gives the label $\mu^{\prime}$ to $t$, the minimal element of the set $N B$.

- Now we need one last lemma.

Lemma 22 As a $G$-module $V \cong \operatorname{ind}_{G^{\prime}}^{G}\left(V\left(x_{n-k+1}, \ldots, x_{n}\right)\right)$.

Proof: For each sequence $\underline{\alpha}=\left(a_{1}, \ldots, a_{k}\right)$ let $\pi_{\underline{\alpha}}$ be the permutation in $G$ which maps $a_{i}$ to $x_{n-k+i}$ and which leaves the elements of $B \backslash\left\{a_{1}, \ldots, a_{k}\right\}$ in increasing order. Then $\pi_{\underline{\alpha}}$ is a set of coset representatives for $G \backslash G^{\prime}$. Since there is one for every $\underline{\alpha}$ this shows that as vector spaces

$$
V \cong V\left(x_{n-k+1}, \ldots, x_{n}\right) \otimes_{G^{\prime}} \mathbb{C}(G) .
$$

Let $g \in G$, let $\left(a_{1}, \ldots, a_{k}\right)=\underline{\alpha}$ be a sequence, and let $b_{i}=g\left(a_{i}\right), \underline{\beta}=\left(b_{1}, \ldots, b_{k}\right)$. Then

$$
\begin{aligned}
& g\left(z_{x_{1}, y_{1}} \wedge \cdots \wedge z_{x_{n-k}, y_{n-k}} \wedge z_{a_{1}, y_{n-k+1}} \wedge \cdots \wedge z_{a_{k}, y_{n}}\right)= \\
& \quad=z_{g\left(x_{1}\right), y_{1}} \wedge \cdots \wedge z_{g_{\left(x_{n-k}\right), y_{n-k}}} \wedge z_{b_{1}, y_{n-k+1}} \wedge \cdots \wedge z_{b_{k}, y_{n}} \\
& \quad=\left(\pi_{\underline{\beta}}^{-1} g \pi_{\underline{\alpha}}\right) z_{x_{1}^{\prime}, y_{1}} \wedge \cdots \wedge z_{x_{n-k}^{\prime}, y_{n-k}} \wedge z_{x_{n-k+1}, y_{n-k+1}} \wedge \cdots \wedge z_{x_{n}, y_{n}}
\end{aligned}
$$

where $x_{i}^{\prime}$ is obtained by replacing the elements of $\left\{x_{a}, \ldots, x_{n}\right\} \backslash\left\{a_{1}, \ldots, a_{k}\right\}$ by the elements of the set $\left\{x_{a}, \ldots, x_{n-k}\right\}$ in order. This computation shows that the vector space isomorphism above is a $G$-module isomorphism.

Now putting all the claims together with the fact $([16])$ that

$$
\operatorname{ind}_{\mathrm{Sym}\left(B^{\prime}\right)}^{\operatorname{Sym}(B)}\left(S^{\mu^{\prime}}\right)=\oplus_{\mathcal{C h}\left(\lambda, \mu^{\prime}\right)} S^{\lambda},
$$

shows that as a module for $G, V$ decomposes as a sum, over all $(X, Y)$ poset tableaux $\Lambda$ of shape $\lambda$, of a copy of $S^{\lambda}$. In the expression 16 we know by induction that 
the Laplacian $L_{Y^{\prime}}$ involving all switches which do not involve the terminal set, i.e., $L_{Y^{\prime}}=L_{Y}-L_{0}$ (where $L_{0}$ is the sum of the switches involving the terminal $Y$-set), acts like the scalar $e_{Y}\left(\Lambda^{\prime}\right)$, where $\Lambda^{\prime}$ is the labeling as far as the point $t$ (the minimal $x$ above $A$ ). The switches involving the terminal $Y$-set $A$ must be studied. But those $y_{i}$ in $A$ can either be switched with each other or with $y_{j}$ that are above an $x_{i} \in B$. By the choice of the terminal set $A, y_{i}$ is comparable to $y_{j}$ for all $y_{i} \in A$. It follows that $L_{0}$ acts on $V=\oplus V\left(a_{1}, \ldots, a_{k}\right)$ by the sum of all transpositions $\left(x_{i}, x_{j}\right)$ for $x_{i} \in B, x_{j} \in\left\{a_{1}, \ldots, a_{k}\right\}$. In terms of our induced module, $L_{0}$ acts on

$$
\operatorname{ind}_{G^{\prime}}^{G}\left(S^{\mu^{\prime}}\right)=S^{\mu^{\prime}} \otimes_{\mathbb{C} G^{\prime}} \mathbb{C} G
$$

like left multiplication on $\mathbb{C} G$ by $\sum_{x_{i}, x_{j} \in B}\left(x_{i}, x_{j}\right)-\sum_{x_{i}, x_{j} \in B^{\prime}}\left(x_{i}, x_{j}\right)$.

But $\sum_{x_{i}, x_{j} \in B^{\prime}}\left(x_{i}, x_{j}\right)$ passes through $\otimes_{\mathbb{C} G^{\prime}}$ to act on $S^{\mu^{\prime}}$ like the scalar $\sum_{x \in \mu^{\prime}} c_{x}$ [24]. And $\sum_{x_{i}, x_{j} \in B}\left(x_{i}, x_{j}\right)$ acts on each $G$-irreducible $S^{\lambda}$ in $S^{\mu^{\prime}} \otimes_{\mathbb{C} G^{\prime}} \mathbb{C} G$ like $\sum_{y \in \lambda} c_{y}$. The result is that $L_{0}$ acts on each copy of $S^{\lambda}$ in $\operatorname{ind}_{G^{\prime}}^{G}\left(S^{\mu^{\prime}}\right)$ like $\sum_{y \in \lambda / \mu^{\prime}} c_{y}$. This explains the scalars $e_{Y}(\Lambda)$ and their multiplicity.

In order to be able to add the eigenvalues of $L_{Y^{\prime}}$ and $L_{0}$, we need the following lemma.

\section{Lemma 23}

$$
L_{0} \cdot L_{Y^{\prime}}=L_{Y^{\prime}} \cdot L_{0}
$$

Proof: Let $\left(x_{i}, x_{j}\right)$ be a transposition in $\operatorname{Sym}(B)$, where $x_{i} \in B^{\prime}$, and let $\left(x_{i}, x_{k}\right)$ be a transposition of $L_{Y^{\prime}}$. By the choice of $B$ we know that $\left(x_{j}, x_{k}\right)$ is also transposition in $L_{Y^{\prime}}$, and since

$$
\left(x_{i}, x_{j}\right)\left(x_{i}, x_{k}\right)=\left(x_{j}, x_{k}\right)\left(x_{i}, x_{j}\right)
$$

the lemma is clear.

- Now we want to consider the case where some of the elements of the sets $A, B$, and $N B=\{$ new $x$ 's $\}$ are equal. So let's write

$$
\begin{aligned}
A & =\alpha_{1} \cup \alpha_{2} \cup \ldots \cup \alpha_{l}, \\
B & =\beta_{1} \cup \beta_{2} \cup \ldots \cup \beta_{m}, \\
N B & =\gamma_{1} \cup \gamma_{2} \cup \ldots \cup \gamma_{n},
\end{aligned}
$$

where $\left|\alpha_{i}\right|=a_{i},\left|\beta_{i}\right|=b_{i}$ and $\left|\gamma_{i}\right|=c_{i}$, and the $y$ 's in each of the $\alpha_{i}$ are equal, the $x$ 's in each of the $\beta_{i}$ are equal, and the $x$ 's in each $\gamma_{i}$ are equal.

Let

$$
\begin{aligned}
\Pi_{A} & =\sum_{\sigma_{i} \in S_{\alpha_{i}}} \sigma_{1} \times \sigma_{2} \times \ldots \times \sigma_{l} \\
\Pi_{B} & =\sum_{\sigma_{i} \in S_{\beta_{i}}}\left(\prod \operatorname{sgn}\left(\sigma_{i}\right)\right) \sigma_{1} \times \ldots \times \sigma_{m} \\
\Pi_{N B} & =\sum_{\sigma_{i} \in S_{\gamma_{i}}}\left(\prod \operatorname{sgn}\left(\sigma_{i}\right)\right) \sigma_{1} \times \ldots \times \sigma_{n} .
\end{aligned}
$$


These projections commute with the Laplacian $L_{Y}$ according to lemma 12 .

Let $\Pi$ be the projection $\Pi=\Pi_{A} \times \Pi_{B} \times \Pi_{N B}$, where the $\Pi_{A}$ acts on the $y$ 's while the other two projections act on the set of the $x$ 's. Then the projection $\Pi$ projects our original space (with all of the $x$ 's and $y$ 's distinct) examined in the previous paragraph to the space where $y_{i}$ 's in each $\alpha_{i}, x$ 's in each $\beta_{i}$ and $\gamma_{i}$ are equal. Each of the components of $\Pi$ commutes with $L_{Y}$ so we know that $\Pi$ and $L_{Y}$ commute.

Lemma 24 The projection $\Pi$ maps the L-block $V$ with all $x$ 's and $y$ 's distinct to the L-block $\Pi V$, where $\alpha_{i}, \beta_{i}$ and $\gamma_{i}$ are sets of equal elements.

Proof: Let $\zeta=z_{x_{1}, y_{1}} \wedge \cdots \wedge z_{a_{1}, y_{i_{1}}} \wedge \cdots \wedge z_{a_{k}, y_{i_{k}}} \wedge \cdots \wedge z_{x_{n}, y_{n}}$. Note that if $y_{i}=y_{j}$, the element $\zeta^{(i, j)}$ and $\zeta$ are the same. In general, if $\sigma_{i} \in S_{\alpha_{i}}$, then $\zeta^{\sigma_{i}}=\zeta$. So we need to identify all equal elements. This is accomplished by projecting with a symmetrizer, i.e., we identify the class of elements $\cup_{\sigma \in S_{\alpha_{i}}} \zeta^{\sigma}$ with the sum $\sum_{\sigma \in S_{\alpha_{i}}} \zeta^{\sigma}$. But $\Pi_{A}$ does exactly this identification, $\Pi_{A}=\sum_{\sigma \in S_{\alpha_{i}}} \zeta^{\sigma}$. The same is true for $\Pi_{B}$ and $\Pi_{N B}$.

NOTE: When we deal with the projection $\Pi_{A}$ there is one important point we have to make. The Laplacian $L_{Y}$ switches all comparable pairs of $y$ 's. If two of the $y$ 's are the same - they would not get switched.

Therefore, when we observed the Laplacian $L_{0}$, we have to subtract all switches involving two $y$ 's from the same $\alpha_{k}$. Each of these transpositions doesn't move any of the $y$ 's (or $x$ 's), and there are exactly $\left(\begin{array}{c}a_{k} \\ 2\end{array}\right)$ of them. Thus, we have to subtract $\left(\begin{array}{c}a_{k} \\ 2\end{array}\right)$ from the eigenvalue $\sum_{y \in \lambda / \mu^{\prime}} c_{y}$ of $L_{0}$.

Thus we can write our space $V$ as a direct sum of the eigenspaces

$$
V=\oplus_{w} V_{w}
$$

where the sum is over all eigenvalues $w$ of $L_{Y}$.

We want to know the eigenvalues of $L_{Y}$ on the image $\Pi V$. We will use that fact that the multiplicity of $w$ as an eigenvalue on $\Pi V$ is the dimension of $\Pi V_{w}$.

So we need to compute the dimension of $\Pi V_{w}$. At present we have $V_{w}$ written in terms of labellings of poset tableaux. So pick such a labeling which at the end has a $\lambda^{\prime}$ at vertex $s$ coming down to a $\mu^{\prime}$ at vertex $t$ then back up to a $\lambda$ at vertex $y_{n}$ (using the notation of this proof). This represents a piece of the eigenspace of the corresponding eigenvalue $w$ where $\hat{G}=\operatorname{Sym}(\hat{B})$ acts like $S^{\lambda^{\prime}}$ and $G^{\prime}=\operatorname{Sym}\left(B^{\prime}\right)$ acts like $S^{\mu^{\prime}}$. We need the following lemma.

Lemma 25 Let $B^{\prime} \subset \hat{B}, N B=\hat{B} \backslash B^{\prime}$. Then the multiplicity of $S^{\mu^{\prime}}$ in $\Pi_{N B} S^{\lambda^{\prime}}$ is equal to the number of ways to get $\mu^{\prime}$ from $\lambda^{\prime}$ by successfully removing vertical strips of lengths $c_{1}, c_{2}, \ldots$ 
Note: As a consequence of this lemma, when we came to an $x$-node $v$ of the repetition number $k(v)$ we have to remove a vertical strip of length $k(v)$.

Proof of lemma: Recall that $N B=\gamma_{1} \cup \gamma_{2} \cup \ldots \cup \gamma_{n}$. The projection $\Pi_{N B}$ projects onto the $\operatorname{sgn}_{\gamma_{1}} \otimes \operatorname{sgn}_{\gamma_{2}} \otimes \cdots \otimes \operatorname{sgn}_{\gamma_{n}}$ isotypic component of $S^{\lambda^{\prime}}$ considered as a $S_{\gamma_{1}} \times \ldots S_{\gamma_{n}}$ module. Thus, for $\mu^{\prime}$ a partition of $\left|B^{\prime}\right|$,

$$
\begin{aligned}
\left\langle\Pi_{N B} S^{\lambda^{\prime}}, S^{\mu^{\prime}}\right\rangle_{S_{B^{\prime}}=}= & \left\langle S^{\lambda^{\prime}}, S^{\mu^{\prime}} \otimes \operatorname{sgn}_{\gamma_{1}} \otimes \cdots \otimes \operatorname{sgn}_{\gamma_{n}}\right\rangle_{S_{B^{\prime}} \times S_{\gamma_{1}} \times \ldots \times S_{\gamma_{n}}} \\
= & \left(\# \text { of ways to get } \mu^{\prime} \text { from } \lambda^{\prime}\right. \text { by successively removing } \\
& \text { vertical strips of lengths } \left.c_{1}, c_{2}, \ldots\right)
\end{aligned}
$$

Now consider the next step of going up from $\mu^{\prime}$ to $\lambda$. At this point we have a piece of the $w$-eigenspace on which $B^{\prime}$ acts like $S^{\mu^{\prime}}$.

The multiplicity coming from this $S^{\mu^{\prime}}$ is $\operatorname{dim}\left(\Pi_{A} \operatorname{ind}_{G^{\prime}}^{G}\left(S^{\mu^{\prime}}\right) \Pi_{B}\right)$. We need to check how $\Pi_{A}$ acts on the induction $\operatorname{ind}_{G^{\prime}}^{G}\left(S^{\mu^{\prime}}\right)=S^{\mu^{\prime}} \otimes_{\mathbb{C} G^{\prime}} \mathbb{C} G$. The $\sigma \in \Pi_{A}$ permutes the $y$ 's. We identified the induction by identifying the sequence of the $x_{i}$ 's that are paired with the set $A$. So switching $y_{i}$ 's has the effect in $\operatorname{Sym}(B)$ of switching the positions corresponding to $B \backslash B^{\prime}$. In other words, $\Pi_{A}-\Pi_{B}$ has the effect of projecting onto the (trivial $\otimes$ sgn $)$ characters of $\left(S_{\alpha_{1}} \times \ldots \times S_{\alpha_{l}}\right) \times\left(S_{\beta_{1}} \times \ldots \times S_{\beta_{m}}\right) \subset S_{B \backslash B^{\prime}} \times S_{B}$ where $S_{B \backslash B^{\prime}} \times S_{B}$ is acting on $S^{\mu^{\prime}} \otimes_{\mathbb{C} G^{\prime}} \mathbb{C} G$ via left multiplication on $\mathbb{C} G$ by $S_{B \backslash B^{\prime}}$ and right multiplication on $\mathbb{C} G$ by $S_{B}$. So to determine $\operatorname{dim}\left(\Pi_{A} \operatorname{ind}_{G^{\prime}}^{G}\left(S^{\mu^{\prime}}\right) \Pi_{B}\right)$ it will be helpful to know the decomposition of the induction $\operatorname{ind}_{G^{\prime}}^{G}\left(S^{\mu^{\prime}}\right)$ as a $S_{B \backslash B^{\prime}} \times S_{B^{-}}$module.

Lemma 26 ([12]) Let $\mu^{\prime} \vdash m, G=S_{r}, G^{\prime}=S_{m}$ and $H=S_{r-m}$ (acting on $\{m+1, \ldots, r\})$. Then as a $H \times G$ module, the induced representation $S^{\mu^{\prime}} \otimes_{\mathbb{C} G^{\prime}} \mathbb{C} G$ decomposes as

$$
\operatorname{ind} d_{G^{\prime}}^{G}\left(S^{\mu^{\prime}}\right)=\oplus_{\lambda \vdash r, \mu \subset \lambda} S^{\lambda / \mu} \otimes S^{\lambda}
$$

Now armed with that lemma, let us return to the dimension count.

$$
\begin{aligned}
\operatorname{dim} & \left(\Pi_{A} \operatorname{ind}_{G^{\prime}}^{G}\left(S^{\mu^{\prime}}\right) \Pi_{B}\right)= \\
= & \left\langle\left(\operatorname{ind}_{G^{\prime}}^{G}\left(S^{\mu^{\prime}}\right)\right) \downarrow,\left(\epsilon_{\alpha_{1}} \otimes \cdots \otimes \epsilon_{\alpha_{l}}\right) \otimes\left(\operatorname{sgn}_{\beta_{1}} \otimes \cdots \otimes \operatorname{sgn}_{\beta_{m}}\right)\right\rangle \\
= & \sum_{\lambda \vdash n, \mu^{\prime} \subset \lambda}\left\langle\left(S^{\lambda / \mu^{\prime}}\right) \downarrow,\left(\epsilon_{\alpha_{1}} \otimes \cdots \otimes \epsilon_{\alpha_{l}}\right)\right\rangle\left\langle S^{\lambda} \downarrow, \operatorname{sgn}_{\beta_{1}} \otimes \cdots \otimes \operatorname{sgn}_{\beta_{m}}\right\rangle \\
= & \sum_{\mu^{\prime} \subset \lambda \vdash n}\left\langle\left(S^{\lambda / \mu^{\prime}} \otimes S^{\lambda}\right) \downarrow,\left(\epsilon_{\alpha_{1}} \otimes \cdots \otimes \epsilon_{\alpha_{l}}\right) \otimes\left(\operatorname{sgn}_{\beta_{1}} \otimes \cdots \otimes \operatorname{sgn}_{\beta_{m}}\right)\right\rangle \\
= & \sum_{\lambda \vdash n}\left(\# \text { of ways to get } \lambda \text { from } \mu^{\prime}\right. \text { by adding horizontal strips of lengths } \\
& \begin{array}{l}
\left.\alpha_{1}, \alpha_{2}, \ldots\right) \cdot(\# \text { of ways to get } \lambda \text { from } \emptyset \text { by removing a vertical } \\
\text { strips of lengths } \left.\beta_{1}, \beta_{2}, \ldots\right)
\end{array}
\end{aligned}
$$


$=\left(\#\right.$ of poset tableaux labellings from $\mu^{\prime}$ up to $\lambda$ then down to $\emptyset$, which add a horizontal strip of length $k$ for every $y$-vertex of repetition number $k$ and subtract a vertical strip of length $k$ for every $x$-vertex of repetition number $k$.)

This completes the proof of the theorem

\subsection{Adding the $L_{X}$}

Consider the Laplacian $L_{X}$. Since we have identified the L-block $V$ with a subspace of the symmetric group algebra $\mathbb{C} S_{n}$, by fixing the order on the $x$ 's, every time the Laplacian $L_{X}$ switches a pair of $x$ 's, it is actually putting a minus sign in front of the corresponding basis element, with the $x$ 's ordered. Since $L_{X}$ acts as a sum of transpositions, every eigenvalue we obtain from the $L_{X}$, will have a minus sign.

Recall the multiplicity of the $x$-node. Let $v$ be an $x$-node of the diagram $P[X, Y]$, labeled with the partition $\Lambda(v)$ and with repetition number $k(v)$. Let $C(v)=\left\{v_{1}, v_{2}, \ldots, v_{l}\right\}$ be the set of covers of $v$. Let $\lambda_{i}$ denote $\Lambda\left(v_{i}\right)$, and let $k_{i}$ denote the repetition numbers, $k\left(v_{i}\right)$. The multiplicity of $\Lambda$ at $v$ is defined to be

$$
m_{v}(\Lambda)=\sum_{\mu} c_{\lambda_{1}, \ldots, \lambda_{l}}^{\mu} c_{\Lambda(v), 1^{k(v)}}^{\mu}
$$

The node-eigenvalue, $e_{v}(\Lambda)$, for each node $v$, is the set of sums of the content over all squares in $\mu / \Lambda(v)$ above for a given $\mu$ minus the binomial coefficient $\left(\begin{array}{c}k(v) \\ 2\end{array}\right)$.

This gives $m_{v}(\Lambda)$ eigenvalues at each $x$-node $v$. We now define the $x$-eigenvalue of $\Lambda$, $e_{x}(\Lambda)$, to be the set of numbers obtained by taking a sum of one element of $e_{v}(\Lambda)$ for each $x$-node $v$. So $\left|e_{x}(\Lambda)\right|=\prod_{x \text {-nodes } v} m_{v}(\Lambda)$.

Theorem 27 ( $L_{X}$-Centerpiece) Let $P$ be a linear poset with a minimum element, $\hat{0}$. Let $X$ and $Y$ be two (multi-)sets, subsets of $P$. For every labeling $\Lambda$ of positive multiplicity, each element in $e_{x}(\Lambda)$ is an eigenvalue of $L_{X}$.

Proof:

The proof of this theorem is similar to the proof of the $L_{Y^{-}}$Centerpiece Theorem. We will omit the details here.

Since $L_{X}$ and $L_{Y}$ commute (as established in Lemma 13 ), the eigenvalues of $L_{X}+L_{Y}$ will be the sum of the eigenvalues on the corresponding irreducibles of the eigenspaces.

Recall that the complete Laplacian $L$ is the sum of three things (from the beginning of this section):

$$
L=L_{D}+L_{X}+L_{Y}
$$

The $L_{D}$ component is the diagonal matrix, which on the L-block $V$ spanned by the sets $(X, Y)$, has value:

$$
e_{D}(X, Y)=w(X, Y)+\Delta(X, Y)
$$




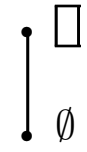

Figure 11: A diagram of the L-block

$$
\begin{aligned}
w(X, Y) & =\sum_{m=1}^{k}\left|\left(x_{m}, y_{m}\right)\right| \\
\Delta(X, Y) & =\sum_{i, j} \delta_{x_{i}, y_{j}}
\end{aligned}
$$

The computer evidence strongly supports the following conjecture:

\section{Complete Centerpiece Conjecture}

Conjecture 28 Let $P$ be a linear poset with a minimum element, $\hat{0}$. Let $X$ and $Y$ be two multisets of vertices of $P$. Let $e_{D}(X, Y)$ be defined as above. For every poset tableau $\Lambda$ of positive multiplicity, let $\tau_{Y}(\Lambda) \in e_{Y}(\Lambda)$ and let $\tau_{X}(\Lambda) \in e_{X}(\Lambda)$. Then the scalars $e(\Lambda)=\tau_{Y}(\Lambda)-\tau_{X}(\Lambda)+e_{D}(X, Y)$ are the complete set of eigenvalues of the Laplacian $L$.

This conjecture claims that the same poset tableau will work simultaneously for both Laplacians $\left(L_{X}\right.$ and $L_{Y}$ ),i.e., that the eigenvalues of the Laplacian $L$ are the sum of the eigenvalues of $L_{Y}$ and the eigenvalues of $L_{X}$ evaluated simultaneously with the same poset tableau.

\section{Homology}

The object of the paper is to get a step closer to evaluating the homology of any Lie algebra corresponding to a linear poset, using only combinatorial properties of the poset. In these two small cases $(n=1$ and $n=2)$ we had no difficulty. For larger $n$, we need some extra results.

\section{$7.1 \quad H_{1}$}

For example, if we want to evaluate the homology $H_{1}\left(L_{P}\right)$ of a Lie algebra $L_{P}$ corresponding to a linear poset $P$, with $\hat{0}$, our construction gives an immediate answer.

An L-block $V$ of size 1 , is determined by the sets $(X, Y), X=\{x\}$, and $Y=\{y\}$. Obviously, if we want $V$ to be non-zero, $x<_{P} y$. So the corresponding diagram of this L-block is given in figure 11.

Both indicators $e_{Y}$ and $e_{X}$ are zero, so the eigenvalues are given by $e_{D}$. But $\Delta(X, Y)=0$ too, since $X \cap Y=\emptyset$. Thus $L\left(z_{x, y}\right)=w(X, Y) z_{x, y}=|(x, y)| z_{x, y}$. In other words, the 


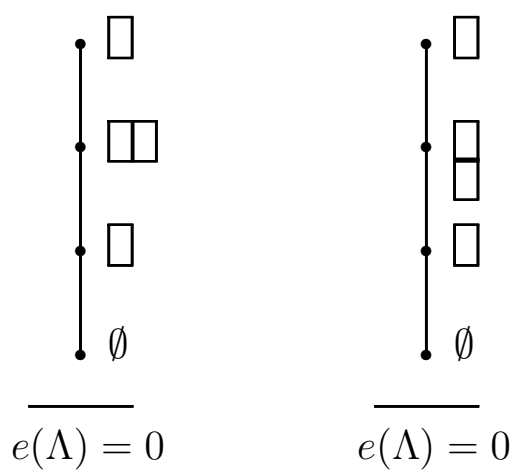

Figure 12: poset tableaux

eigenvectors of the Laplacian $L_{1}$ are the basis vectors $z_{x, y}$, and the corresponding eigenvalues are $|(x, y)|$, i.e., the number of the vertices in the poset $P$, between $x$ and $y$.

The dimension of the homology is the number of zero eigenvalues, i.e., the number of the intervals $z_{x, y}$, such that $y$ covers $x$.

Thus

$$
\operatorname{dim}\left(H_{1}\left(L_{P}\right)\right)=(\# \text { of covering relations in } P)
$$

\section{$7.2 \quad H_{2}$}

In this case the L-block $V$ in question is spanned by the (multi-)sets $(X, Y)$, each of size 2, i.e., $X=\left\{x_{1}, x_{2}\right\}$ and $Y=\left\{y_{1}, y_{2}\right\}$. There are several possibilities for the L-block.

1. All four elements are comparable, and $x$ 's are below the $y$ 's.

$$
x_{1}<x_{2}<y_{1}<y_{2}
$$

All possible poset tableaux are shown in figure 12 .

As we can see, both $e_{Y}$ and $e_{X}$ eigenvalues are zero. So we don't have to worry how to add them up - we will always get zero. $\Delta$ is also zero, since the sets $X$ and $Y$ are disjoint. Thus again, the Laplacian is $L\left(z_{x_{1}, y_{1}} \wedge z_{x_{2}, y_{2}}\right)=w(X, Y)\left(z_{x_{1}, y_{1}} \wedge z_{x_{2}, y_{2}}\right)$. But in this case, both intervals contain at least one element, so $w(X, Y)>0$. Thus in this case we never get a zero eigenvalue, which might contribute to the homology $\mathrm{H}_{2}$.

2. y's are not comparable.

$$
x_{1}<x_{2}<\begin{aligned}
& y_{1} \\
& y_{2}
\end{aligned}
$$

All possible poset tableaux are shown in figure 13.

The value of $e_{Y}$ is zero, while the values of $e_{X}$ are +1 and -1 . Since $e_{D}$ is always non-negative, the value +1 cannot contribute to homology $H_{2}$. The other value can, but only if $e_{D}$ is +1 . That means that all of the relations indicated: $x_{1}<x_{2}, x_{2}<y_{1}$ and $x_{2}<y_{2}$ are covering relations in the poset $P$. Whenever we have a four-element 


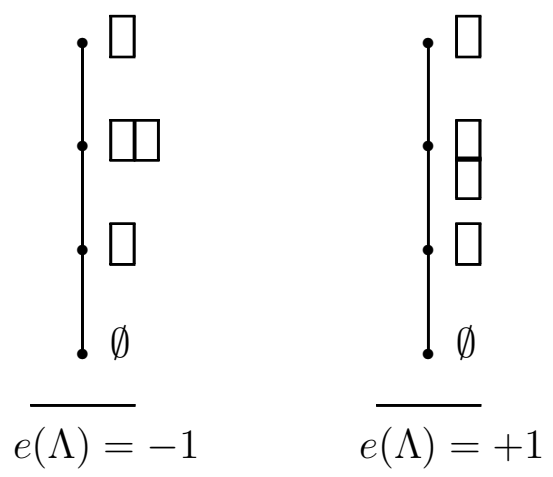

Figure 13: poset tableaux

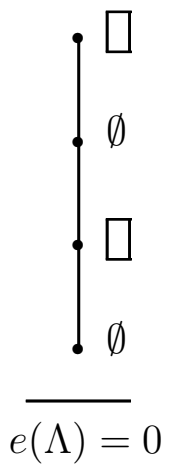

Figure 14: poset tableau

subset of the poset $P$, with covering relations $x_{1}<x_{2}, x_{2}<y_{1}$ and $x_{2}<y_{2}$, we will call that a "Y"-configuration. Thus in this case every occurrence of "Y"-configuration (described as above) in the Hasse diagram of the poset contributes to the homology $\mathrm{H}_{2}$.

3. $x_{1}<y_{1}<x_{2}<y_{2}$ or equivalently (for our purpose) $x_{1}<y_{1}=x_{2}<y_{2}$.

There is only one poset tableau spanned by these sets $X$ and $Y$, namely the one shown in figure 14 .

Since the space is one dimensional, we will add the eigenvalues in the only possible way. In the first case, the eigenvalue will be zero, if and only if both relations, $x_{1}<y_{1}$ and $x_{2}<y_{2}$, are covering relations, i.e., every occurrence of a distinct (all vertices are distinct) pair of covering relations contributes to the dimension of the homology $H_{2}$. If the second case occurs, i.e., if $y_{1}=x_{2}$ then $\Delta$ will contribute to the eigenvalue, and it won't be zero anymore.

4. $x_{1}<x_{2}<y_{1}=y_{2}$.

There is only one poset tableau spanned by these sets $X$ and $Y$, namely the one shown on the figure 15 . 


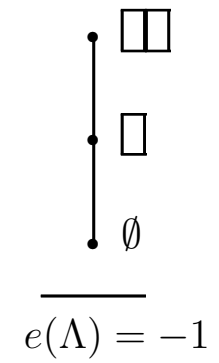

Figure 15: poset tableau

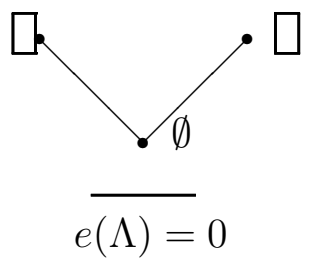

Figure 16: poset tableau

Since the space is one dimensional, we will add the eigenvalues in the only possible way. The eigenvalue will be zero, if and only if $x_{2}$ covers $x_{1}$, and both $y_{1}$ and $y_{2}$ cover $x_{2}$. Note that this is a degenerate letter "Y", with a joint top vertex. Every such occurrence contributes to the homology $\mathrm{H}_{2}$.

5. $x$ 's are the same.

$$
x_{1}=x_{2}<\begin{aligned}
& y_{1} \\
& y_{2}
\end{aligned} .
$$

There is only one poset tableau spanned by these sets $X$ and $Y$, namely the one shown in figure 16.

Since the space is one dimensional, we will add the eigenvalues in the only possible way. The eigenvalue will be zero, if and only if both $y_{1}$ and $y_{2}$ cover $x_{1}=x_{2}$. Again, it is a pair of distinct covering relations with joint vertex, this time the $x$-vertex. Every such occurrence contributes to the homology $\mathrm{H}_{2}$.

6. $x_{1}=x_{2}<y_{1}<y_{2}$. There is only one poset tableau spanned by these sets $X$ and $Y$, namely the one shown on the figure 17 .

Since the space is one dimensional, we will add the eigenvalues in the only possible way. Since $e_{D}$ is always non-negative, the value +1 cannot contribute to homology $\mathrm{H}_{2}$.

7. The trivial case when $x$ 's are not comparable (so because of linearity and the minimum element neither are $y$ 's).

$$
\begin{aligned}
& x_{1}<y_{1} \\
& x_{2}<y_{2}
\end{aligned} .
$$




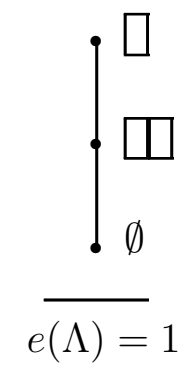

Figure 17: poset tableau

This is equivalent to case 3 . We have two distinct covering relations, so it contributes to the homology $\mathrm{H}_{2}$.

All together, the dimension of the homology $\mathrm{H}_{2}$ is in fact the number of distinct pairs of covering relations + the number of occurrences of letter " $\mathrm{Y}$ " in the poset $P$. In other words, if $\mathcal{H}$ is a Hasse diagram of a poset $P$, with $e$ edges, and $\gamma$ letter "Y"'s (degenerate or not), we have $H_{1}\left(L_{P}\right)=\mathbb{C}^{e}$ and $H_{2}=\mathbb{C}^{\left(\begin{array}{l}e \\ 2\end{array}\right)+\gamma}$.

\subsection{Examples}

- For example, suppose that we are dealing with the chain poset on $n$ vertices $(1<2<$ $\cdots<n), T_{n}$. The number of non-degenerate Y's is zero. The number of degenerate letters $\mathrm{Y}$ is $(\mathrm{n}-2)$. The number of edges in the Hasse diagram is (n-1). Hence

$$
H_{1}\left(T_{n}\right)=\mathbb{C}^{n-1}
$$

and

$$
H_{2}\left(T_{n}\right)=\mathbb{C}^{n-2+\left(\begin{array}{c}
n-1 \\
2
\end{array}\right)}=\mathbb{C}^{\frac{(n+1)(n-2)}{2}}
$$

- Let the poset $P$ be given in figure 18, where the length of the chains are $m$ and $n$. This is in fact equivalent to having two disjoint chains, of length $m$ and $n$. Thus the corresponding homologies will be $H_{1}=\mathbb{C}^{n-1+m-1}$, and $H_{2}\left(T_{n}\right)=\mathbb{C}^{\frac{(n+1)(n-2)}{2}+\frac{(m+1)(m-2)}{2}}$

\section{Conclusion}

These results have several interesting corollaries that are of a combinatorial nature. We will state one. Let $P$ be a rooted tree on $n$ nodes and let $\Sigma$ be the sum in the group algebra of $S_{n}$ of all transpositions $(i, j)$ such that $i$ is on the unique path from $j$ to the root in $P$. Then $\Sigma$ acting on $\mathbb{C} S_{n}$ by left multiplication has non-negative integer eigenvalues and the corresponding eigenspaces can be identified in representation-theoretic terms.

There is still a lot to do in this area. Although my work provides partial answers and a conjecture for all rooted trees, the question is still open for other posets. What 


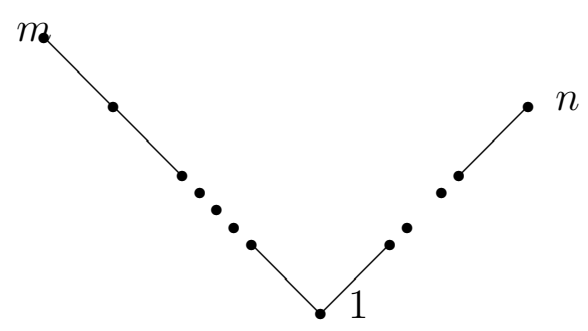

Figure 18: poset $P$

happens in those cases is very difficult to control, since the expression for the Laplacian becomes more complicated. In the tree case I wish to examine a twisting of the Laplacian by a parameter $\alpha$ which, my advisor has shown, is related to Jack polynomials and the Krawtchouk polynomials in certain special cases. Lastly I would like to see if the more algebraic consequences of Kostant's theorem have sensible analogues in my case.

Acknowledgement. I would like to thank my advisor, Professor Phil Hanlon, for the guidance, help, and for his invaluable suggestions.

\section{References}

[1] K. Baclawski, Cohen-Macaulay ordered sets, J. Algebra, [63] (1980), 226-258.

[2] A. Björner, On the homology of geometric lattices, Algebra Universalis, [14] (1982), 107-128.

[3] A. Björner, A. M. Garsia, R. P. Stanley, An introduction to Cohen-Macaulay partially ordered sets, Ordered Sets (I. Rival ed.), 563-615, Boston: D. Reidel Publishing Co., 1981.

[4] H. Cartan, S. Eilenberg, Homological algebra, Princeton University Press, Princeton, 1956.

[5] H. H. Crapo, The Möbius function of a lattice, Journal of Comb. Theory, [1] (1966), 126-131.

[6] C. Chevalley, S. Eilenberg, Cohomology theory of Lie groups and Lie algebras, Trans. of the AMS, [63] (1948), 85-124.

[7] Y. Chow, General theory of Lie algebras, Gordon and Breach, New York, 1978.

[8] F. D. Farmer, Cellular homology for posets, Math. Japonica, [23] (1979), 607-613. 
[9] D. B. Fuks, Cohomology of infinite-dimensional Lie algebras, Consultants Bureau, New York, 1986.

[10] M. Gerstenhaber, S.D. Schack, The cohomology of presheaves of algebras I., Trans. of the AMS, [310] (1988), no.1, 135-165.

[11] G. P. Hochschild, J. P. Serre, Cohomology of Lie algebras. Ann. of Math., [57] (1953), 591-603.

[12] P. Hanlon, Some twisted random walks indexed by sequences, preprint, (1993).

[13] G. P. Hochschild, Basic theory of algebraic groups and Lie algebras, Springer-Verlag, New York, 1981.

[14] J. E. Humphreys, Introduction to Lie algebras and representation theory, SpringerVerlag, New York, 1972.

[15] N. Jacobson, Lie algebras, Dover Publications Inc., New York, 1962.

[16] G. D. James, The representation theory of the symmetric group, Springer-Verlag, Berlin Heidelberg New York, 1978.

[17] G. James, A. Kerber, The representation theory of the symmetric group, AddisonWesley Publishing Company, Reading, MA, 1981.

[18] A. W. Knapp, Lie groups, Lie algebras, and cohomology, Princeton University Press, Princeton, 1988.

[19] B. Kostant, Lie algebra cohomology and the generalized Borel-Weil theorem, Ann. of Math., [74] (1961), 329-387.

[20] B. Kostant, Lie algebra cohomology and generalized Schubert cells, Ann. of Math., [77] (1963), 72-144.

[21] J. L. Koszul. Homologie et cohomologie des algèbres de Lie, Bull. Soc. Math. France, [78] (1950), 65-127.

[22] H. Lakser, The homology of lattice, Discrete Math., [1] (1971), 187-192.

[23] G. Leger, E. Luks, Cohomology and weight systems for nilpotent Lie algebras, Bull. Amer. Math. Soc., [80] (1974), 77-80.

[24] I. G. Macdonald, Symmetric functions and Hall polynomials, Oxford University Press, New York, 1979.

[25] J. Mather, Invariance of the homology of a lattice, Proc. Amer. Math. Soc., [17] (1966), 1120-1124.

[26] G. E. Murphy, A new construction of Young's seminormal representation of the symmetric group, Journal of Algebra, [69] (1981), 287-297. 
[27] M. H. Peel, Specht modules and symmetric groups, Journal of Algebra, [36] (1975), 88-97.

[28] G. de B. Robinson, Representation theory of the symmetric group, University of Toronto Press, 1961.

[29] G.-C. Rota, On the foundations of combinatorial theory. I: Theory of Möbius functions, Z. Wahrsch. Verw. Gebiete, [2] (1964), 340-368.

[30] B. E. Sagan, The symmetric group, Wadsworth \& Brooks/Cole, Advanced Books \& Software, Pacific Grove, California, 1991.

[31] R. P. Stanley, Enumerative combinatorics, v. 1, Wadsworth and Brooks/Cole Mathematics Series, Monterey, California, 1986.

[32] G. P. Thomas, On Schensted's construction and the multiplication of Schur functions, Advances in Math., [30] (1978), 8-32.

[33] J. Walker, Homotopy type and Euler characteristic of partially ordered sets, European J. Combinatorics, [2] (1981), 373-384.

[34] D. E. White, Some connections between the Littlewood-Richardson rule and the construction of Schensted, Journal of Comb. Theory, Series A, [30] (1981), 237-247. 